$\begin{array}{ll}\text { Research Square } & \begin{array}{l}\text { Preprints are preliminary reports that have not undergone peer review. } \\ \text { They should not be considered conclusive, used to inform clinical practice, } \\ \text { or referenced by the media as validated information. }\end{array}\end{array}$

\title{
Global Identification of C2 Domain Contain Proteins And Characterization Of Genetic Variations For Involved Abiotic Stress Tolerance In Rice (Oryza sativa L.)
}

Hongjia Zhang

Department of Plant Bioscience, College of Natural Resources and Life Science, Pusan National University, Miryang 50463

Yuting Zeng

College of Agricultural, Yangtze university, Jingzhou, 434000

\section{Jeonghwan Seo}

Life and Industry Convergence Research Institute, Pusan National University, Miryang 50463

Yu-Jin Kim

Department of Life Science \& Environmental Biochemistry, College of Natural Resources and Life Science, Pusan National University, Miryang 50463

\section{Sun Tae Kim}

Department of Plant Bioscience, College of Natural Resources and Life Science, Pusan National University, Miryang 50463

Soon-Wook Kwon ( $\square$ swkwon@pusan.ac.kr)

Department of Plant Bioscience, College of Natural Resources and Life Science, Pusan National University, Miryang 50463

\section{Research Article}

Keywords: Rice, C2 domain contain protein (OsC2DP), Abiotic stress, Haplotype, Phylogenetics, Expression pattern

Posted Date: July 12th, 2021

DOI: https://doi.org/10.21203/rs.3.rs-618284/v1

License: () (1) This work is licensed under a Creative Commons Attribution 4.0 International License. Read Full License 


\section{Abstract}

\section{Background}

C2DPs (C2 domain contain proteins) have been identified in different genomes that contain single or multiple $\mathrm{C} 2$ domains in their $\mathrm{C}$ or $\mathrm{N}$-terminal, it possesses higher functional activity in the cell membrane between the cytoplasm and nucleus. Despite the identification of MCTPs and NTMC2s in rice, Arabidopsis, and cotton in a previous study, however, the $C 2 D P$ gene family in rice has not been comprehensively studied, and the role of the $C 2 D P$ gene in rice in response to abiotic stress is unclear.

\section{Results}

In this study, we identified 82 C2DPs in the rice genome and divided them into seven groups through phylogenetic analysis. Synteny analysis revealed that duplication events were either exhibited within the genome of rice or between the genome of rice and other species. Through the analysis of cis-acting elements in promoters, expression profiles, and qRT-PCR results, the functions of OsC2DPs were found to be widely expressed in diverse tissues and were extensively involved in phytohormones and abiotic stress in rice. Prediction of the miRNA targets of OsC2DPs revealed that some of the homolog genes were regulated by consistent miRNAs and may carry out redundancy function. Notably, OsC2DP50/51/52 as a co-tandem duplication exhibited similar expression variations and involved the coincident miRNA-regulation pathway. Moreover, the results of SNP genotyping and haplotype analysis revealed that OSC2DP17, OsC2DP29, and OsC2DP49 possessed diverse haplotypes for impacted cold tolerance owing to genomic variations.

\section{Conclusions}

These findings provide a comprehensive sight for characterized OsC2DPs in rice and their roles for abiotic stress. Further, the genetic variation supports the theoretical reference for molecular breeding in rice.

\section{Background}

$\mathrm{Ca}$ is a necessary factor for plant development as it serves as a signaling factor for transduction functions in multiple physiological processes [1]. Ca maintains the steadiness of the cell wall, membrane, and membrane-binding protein and improves the resistance to diverse abiotic and biotic stresses in plant cells, thereby triggering multiple signaling pathways [2,3]. Ca-binding protein is a kind of protein for specific binding to the intracellular free Ca2+, the signaling transduction function made by binding for each other. To date, 3 major Ca-binding proteins, namely calmodulins [4], Ca-dependent protein kinase [5], calcineurin B-like proteins [6], have been identified as well-known $\mathrm{Ca}^{2+}$ sensors.

C2DPs, a type of Ca-dependent protein kinase, are highly conserved long term. The typical C2 domain consists of approximately 135 amino acids and was given the name, PKC-C2 (PFAM: PF00168), in a previous study [7]; the other two branches of the C2 domain super family include the PI3K-C2 (PFAM:

PF00792) and PTEN-C2 domains (PF10409). Most PKC-C2 proteins are involved in $\mathrm{Ca}^{2+}$-dependent interactions with the membrane and most previous reports discussed PKC-C2 [8]. Generally, C2DPs contain at least one C2 domain, with or without transmembrane domains in the C-terminus of the protein. However, several C2 domains are frequently coupled to other enzymatic domains, such as the phospholipase domain [9, 10], synaptotagmin domain [11], and phosphatidylinositol domain [12], which bind to the membrane or point to other functions. With the development of protein structure, a set of multiple C2 domains and transmembrane region proteins were identified as branches of C2DPs [13], which contained 3-4 C2 domains at the N-terminus and 1-4 transmembrane regions at the $\mathrm{C}$ terminus. In membrane transport proteins, multiple $\mathrm{C} 2$ domains have been found to have different but conserved sequences, suggesting the formation of more biological functions due to the cooperation or crosstalk between multiple C2 domains [14].

Related studies on C2DP started from a long time before in eukaryon. The first characterized C2 domain was found in mammalian cells, the animal PKC contained 3-4 conserved domains, named C1, C2, C3, and C4, respectively. Interestingly, C1, C3, and C4 exist in all PKCs; only the C2 domain exists in Ca ${ }^{2+}$ dependent PKC, indicating a synergistic relationship between the $\mathrm{Ca}^{2+}$ and $\mathrm{C} 2$ domains [15]. The function of the $\mathrm{C} 2$ domain has also been reported [16, 17]. The $\mathrm{C} 2$ domain mediates the transduction mechanism whereby peptides derived from PKC modulate protein-protein interactions within PKC and the PKC binding proteins [18]. The peptides derived from the $\mathrm{C} 2$ domain were also identified as two branches, isozyme-selective activator and selective inhibitor of epsilon PKC, which play important roles in the prevention and protection of cardiac and brain ischemic damage, diabetes complications, and transplanted hearts [19].

The functions of C2DPs have also been reported in plants within the last 20 years [20,21, 22]. In Arabidopsis thaliana, gene SYT1, which belongs to Synaptotagmins, was identified by the affected development of the cell. Synaptotagmins constitute a family by characterized of $\mathrm{N}$-terminal transmembrane region and two $\mathrm{C} 2$ domains at the $\mathrm{C}$-terminal. SYT1, which is widely expressed and is located in the plasma membrane, has all the characterized domains of Synaptotagmins. However, when the function of SYT1 is repressed, the plasma membrane has reduced integrity, which decreases cell viability [11]. The $Q K Y$ gene has four predicted C2 domains and is the first characterized MCTP in Arabidopsis. qky mutant plants showed defects in the development of the outer integument, floral organ, and stem, and impacted the growth of floral meristem and root hair. QKY can bind with the receptor-like kinase, SUB, to coordinate the regulation of intercellular communication and organ development [23]. In rice, OsERG1, which is the first reported C2DP, expression of OsERG1 increased following treatment of Magnaporthe grisea infection and $\mathrm{Ca}^{2+}$ ions, the subcellular localization from the cytoplasm to the cell membrane, and participates in the plant defense signaling pathway [24]. OsSMCP1, a $\mathrm{Ca}^{2+}$-dependent membrane-targeting domain protein, contains a single $\mathrm{C} 2$ domain. Overexpression of OsSMCP1 enhances plant tolerance to salt, drought, osmotic, and oxidative stress, and causes improved resistance to Pseudomonas syringae [25]. OsC2DP (LOC_Os09g39770) is also significantly involved in salt tolerance in rice. The OsC2DP CRISPR/Cas9 knockout mutant line was found to be more sensitive to salt stress than WT, and salt treatment caused the translocation of OsC2DP from the cytoplasm to the cell membrane [26], similar to the shift identified for the protein, OsERG1 [24]. To date, gene family analysis of the $\mathrm{C} 2$ domain has only been reported for few species, in Arabidopsis and cotton, the study revealed 
results for MCTPs, a branch of the C2DP family, divided into five subfamilies according to the phylogenetic tree [27, 28]. In rice, a comprehensive analysis of NTMC2s revealed 13 family members divided into six subfamilies according to phylogeny and motif constitution [29]. Therefore, the C2DP gene family of rice requires further comprehensive analysis to identify the relationships and functions of the members.

In this study, 82 family members of the C2DP family were identified in rice, named the OSC2DP gene family. In addition, seven subgroups were identified based on homologous relationships and conserved domain structures. Our results provide a theoretical basis for the future characterization of the roles of OsC2DPs in abiotic stress in rice.

\section{Results}

\section{Identification, polygenetic, and sequence analyses of OsC2DPs}

To identify the OsC2DP gene family members in rice, a BLAST search was performed for the rice genome using the HMM model (PF00168) and the C2domain reconfirmed in the InterPro and SMART databases. As a result, 82 OsC2DPs were identified by filtering an E-value $<1 \mathrm{e}^{-5}$ (Additional file 1: Table S1). Whole OsC2DPs were found to have different basic characteristics (Table 1). Further, the genome DNA length ranged from 784 bp (OsC2DP67) to 13170 bp (OsC2DP62), with an average of $4484 \mathrm{bp}$, while the cds length ranged from $432 \mathrm{bp}$ (OsC2DP20) to $6354 \mathrm{bp}$ (OsC2DP80), with an average of 1875 bp. The physical properties were also revealed to be widespread (Table 1). The pl of OsC2DPs ranged from 4.22 (OsC2DP36) to 11.23 (OsC2DP12), with an average of 7.13; 46 members had $\mathrm{pl}<6$ while 31 members had pl > 8. Further, the Mw of OsC2DPs ranged from $8.847 \mathrm{kD}$ (OsC2DP54) to 227.943 kD (OsC2DP80), with an average of $65.62 \mathrm{kD}$. The chromosome distribution results showed that OsC2DPs were present in each chromosome (Additional file 2: Fig. S1), with most chromosomes existing in many members, except chr08, chr10, and chr11. Interestingly, there were few gene clusters in the closed position, such as 0sC2DP7 and OsC2DP8 in chr01, and OsC2DP67 and OsC2DP68 in chr07. Further, a larger cluster was identified in chr06, which included OsC2DP50 to OsC2DP57, implying that unknown tandem or segmental duplications might exist in a few regions. We also analyzed the prediction of subcellular localization of OsC2PDs and found that the OsC2DPs performed functions in multiple positions, including nuclear, chloroplast, cytoplasmic, membrane, and mitochondrial (Additional file 1: Table S2), and most members were found to gather in the cytoplasm and nucleus (Additional file 2: Fig. S2). 
Table 1

Gene list and information for OsC2DP gene family

\begin{tabular}{|c|c|c|c|c|c|c|c|c|c|}
\hline Gene Symbol & RAP-ID & MSU-ID & Chr. & Start & End & Gene Length & Cds Length & PI & MW \\
\hline OsC2DP1 & Os01g0128800 & LOC_Os01g03820 & Chr1 & 1597486 & 1604610 & 7125 & 1956 & 7.71 & 66142.35 \\
\hline OsC2DP2 & Os01g0172400 & LOC_Os01g07760 & Chr1 & 3724314 & 3729284 & 4971 & 2439 & 5.6 & 92219.32 \\
\hline OsC2DP3 & Os01g0242600 & LOC_Os01g14050 & Chr1 & 7862001 & 7867812 & 5812 & 2025 & 5.82 & 75308.13 \\
\hline OsC2DP4 & Os01g0369500 & LOC_Os01g27190 & Chr1 & 15160423 & 15161640 & 1218 & 1020 & 6.83 & 31498.49 \\
\hline OsC2DP5 & Os01g0587300 & LOC_Os01g40480 & Chr1 & 22868546 & 22872149 & 3604 & 3243 & 7.42 & 119356.5 \\
\hline OsC2DP6 & Os01g0819150 & LOC_Os01g60340 & Chr1 & 34902303 & 34906955 & 4653 & 1563 & 8.49 & 50328.86 \\
\hline OsC2DP7 & Os01g0819300 & LOC_Os01g60350 & Chr1 & 34908079 & 34912602 & 4524 & 1548 & 5.75 & 58441.86 \\
\hline OsC2DP8 & Os01g0841700 & LOC_Os01g62430 & Chr1 & 36135811 & 36137295 & 1485 & 570 & 6.04 & 17735.82 \\
\hline OsC2DP9 & Os01g0853800 & LOC_Os01g63470 & Chr1 & 36770986 & 36772435 & 1450 & 984 & 9.1 & 35355.71 \\
\hline OsC2DP10 & Os01g0934100 & LOC_Os01g70790 & Chr1 & 40973255 & 40974782 & 1528 & 1077 & 8.71 & 36238.43 \\
\hline OsC2DP11 & Os01g0951100 & LOC_Os01g72230 & Chr1 & 41882488 & 41886410 & 3923 & 1146 & 8.52 & 42706.69 \\
\hline OsC2DP12 & Os02g0120200 & LOC_Os02g02790 & Chr2 & 1061877 & 1066213 & 4337 & 2733 & 11.23 & 99144.34 \\
\hline OsC2DP13 & Os02g0198300 & LOC_Os02g10480 & Chr2 & 5505016 & 5508425 & 3410 & 963 & 5.51 & 35250.87 \\
\hline OsC2DP14 & Os02g0199800 & LOC_Os02g10630 & Chr2 & 5586939 & 5593645 & 6707 & 3336 & 8.18 & 123146.8 \\
\hline OsC2DP15 & Os02g0313700 & LOC_Os02g20970 & Chr2 & 12398421 & 12404925 & 6505 & 2217 & 6.07 & 80965.85 \\
\hline OsC2DP16 & Os02g0327000 & LOC_Os02g22130 & Chr2 & 13173639 & 13176606 & 2968 & 498 & 5.95 & 18547.61 \\
\hline OsC2DP17 & Os02g0448400 & LOC_Os02g25060 & Chr2 & 14529662 & 14537548 & 7887 & 1617 & 6.36 & 61026.85 \\
\hline OsC2DP18 & Os02g0521300 & LOC_Os02g32160 & Chr2 & 18994014 & 19001080 & 7067 & 1755 & 5.26 & 63830.39 \\
\hline OsC2DP19 & Os02g0605400 & LOC_Os02g39280 & Chr2 & 23720437 & 23726180 & 5744 & 1578 & 9.15 & 50703.85 \\
\hline OsC2DP20 & Os02g0640000 & LOC_Os02g42710 & Chr2 & 25684044 & 25687149 & 3106 & 432 & 4.56 & 15775.67 \\
\hline OsC2DP21 & Os02g0663900 & LOC_Os02g44490 & Chr2 & 26924717 & 26927173 & 2457 & 2340 & 9.12 & 88984.9 \\
\hline OsC2DP22 & Os02g0665100 & LOC_Os02g44560 & Chr2 & 27009020 & 27010017 & 998 & 666 & 9.15 & 25491.47 \\
\hline OsC2DP23 & Os02g0722450 & LOC_Os02g49070 & Chr2 & 29997568 & 30002813 & 5246 & 999 & 5.58 & 36843.03 \\
\hline OsC2DP24 & Os02g0815100 & LOC_Os02g57000 & Chr2 & 34920542 & 34921736 & 1195 & 858 & 9.82 & 30640.46 \\
\hline OsC2DP25 & Os02g0816000 & LOC_Os02g57090 & Chr2 & 34958012 & 34961608 & 3597 & 3000 & 9.42 & 110780.7 \\
\hline OsC2DP26 & Os02g0829200 & LOC_Os02g58230 & Chr2 & 35643004 & 35648165 & 5162 & 1977 & 6.54 & 72271.9 \\
\hline OsC2DP27 & Os03g0119100 & LOC_Os03g02740 & Chr3 & 1027577 & 1031646 & 4070 & 2715 & 7.04 & 100864.2 \\
\hline OsC2DP28 & Os03g0194100 & LOC_Os03g09840 & Chr3 & 4894522 & 4897057 & 2536 & 1209 & 4.54 & 43864.28 \\
\hline OsC2DP29 & Os03g0251600 & LOC_Os03g14700 & Chr3 & 7989864 & 7994679 & 4816 & 1815 & 6.14 & 65065.62 \\
\hline OsC2DP30 & Os03g0289300 & LOC_Os03g18010 & Chr3 & 10025401 & 10027931 & 2531 & 1455 & 6.04 & 67382.87 \\
\hline OsC2DP31 & Os03g0391400 & LOC_Os03g27370 & Chr3 & 15673720 & 15679214 & 5495 & 2556 & 8.31 & 93550.93 \\
\hline OsC2DP32 & Os03g0652000 & LOC_Os03g44890 & Chr3 & 25326521 & 25330063 & 3543 & 3165 & 8.73 & 115492 \\
\hline OsC2DP33 & Os03g0840800 & LOC_Os03g62410 & Chr3 & 35350196 & 35354897 & 4702 & 2544 & 7.63 & 60988.55 \\
\hline OsC2DP34 & Os04g0472900 & LOC_Os04g39680 & Chr4 & 23640853 & 23643655 & 2803 & 2568 & 9.51 & 88152.69 \\
\hline OsC2DP35 & Os04g0476600 & LOC_Os04g40070 & Chr4 & 23859220 & 23863737 & 4518 & 2121 & 5.88 & 79244.91 \\
\hline OsC2DP36 & Os04g0531100 & LOC_Os04g44870 & Chr4 & 26558810 & 26561326 & 2517 & 435 & 4.22 & 15868.54 \\
\hline OsC2DP37 & Os04g0644900 & LOC_Os04g55220 & Chr4 & 32826758 & 32831122 & 4365 & 1728 & 6.99 & 65016.4 \\
\hline OsC2DP38 & Os04g0682100 & LOC_Os04g58570 & Chr4 & 34824425 & 34826306 & 1882 & 438 & 4.58 & 16107.97 \\
\hline OsC2DP39 & Os04g0683800 & LOC_Os04g58720 & Chr4 & 34915791 & 34920089 & 4299 & 3036 & 9.1 & 114912.4 \\
\hline OsC2DP40 & Os04g0691800 & LOC_Os04g59520 & Chr4 & 35398604 & 35402604 & 4001 & 3066 & 9.31 & 113276.4 \\
\hline OsC2DP41 & Os05g0127200 & LOC_Os05g03610 & Chr5 & 1535708 & 1541571 & 5864 & 1797 & 5.69 & 67052.3 \\
\hline
\end{tabular}




\begin{tabular}{|c|c|c|c|c|c|c|c|c|c|}
\hline Gene Symbol & RAP-ID & MSU-ID & Chr. & Start & End & Gene Length & Cds Length & PI & MW \\
\hline OsC2DP42 & Os05g0149100 & LOC_Os05g05650 & Chr5 & 2806163 & 2808004 & 1842 & 840 & 9.42 & 28864.57 \\
\hline OsC2DP43 & Os05g0171000 & LOC_Os05g07880 & Chr5 & 4255763 & 4260333 & 4571 & 2475 & 6.13 & 76484.67 \\
\hline OsC2DP44 & Os05g0370600 & LOC_Os05g30750 & Chr5 & 17812073 & 17816465 & 4393 & 2325 & 9.21 & 89345.46 \\
\hline OsC2DP45 & Os05g0373300 & LOC_Os05g30970 & Chr5 & 17983282 & 17989911 & 6630 & 1791 & 5.3 & 59904.44 \\
\hline OsC2DP46 & Os05g0382000 & LOC_Os05g31720 & Chr5 & 18466963 & 18470649 & 3687 & 1188 & 5.91 & 44599.41 \\
\hline OsC2DP47 & Os05g0429700 & LOC_Os05g35480 & Chr5 & 21080988 & 21084058 & 3071 & 2415 & 9.5 & 90463.05 \\
\hline OsC2DP48 & Os06g0223800 & LOC_Os06g11990 & Chr6 & 6401419 & 6407785 & 6367 & 3378 & 5.27 & 105945.3 \\
\hline OsC2DP49 & Os06g0297800 & LOC_Os06g19400 & Chr6 & 11046380 & 11054423 & 8044 & 3114 & 5.45 & 115810 \\
\hline OsC2DP50 & Os06g0604200 & LOC_Os06g40170 & Chr6 & 23908918 & 23913797 & 4880 & 2499 & 5.92 & 92513.68 \\
\hline OsC2DP51 & Os06g0604300 & LOC_Os06g40180 & Chr6 & 23921940 & 23924921 & 2982 & 2529 & 5.89 & 93236.21 \\
\hline OsC2DP52 & Os06g0604400 & LOC_Os06g40190 & Chr6 & 23928702 & 23932372 & 3671 & 2454 & 6.56 & 91318.9 \\
\hline OsC2DP53 & Os06g0607900 & LOC_Os06g40570 & Chr6 & 24187249 & 24192151 & 4903 & 3012 & 9.04 & 40376.51 \\
\hline OsC2DP54 & Os06g0609450 & LOC_Os06g40704 & Chr6 & 24264404 & 24268015 & 3612 & 966 & 9.59 & 8847.43 \\
\hline OsC2DP55 & Os06g0614000 & LOC_Os06g41090 & Chr6 & 24555313 & 24557973 & 2661 & 2475 & 9.24 & 92947.99 \\
\hline OsC2DP56 & Os06g0638900 & LOC_Os06g43190 & Chr6 & 25963873 & 25967411 & 3539 & 927 & 8.57 & 34271.57 \\
\hline OsC2DP57 & Os06g0685300 & LOC_Os06g47130 & Chr6 & 28558292 & 28564762 & 6471 & 1623 & 9.15 & 11160.86 \\
\hline OsC2DP58 & Os07g0108400 & LOC_Os07g01770 & Chr7 & 452093 & 460151 & 8059 & 1650 & 9.07 & 18645.64 \\
\hline OsC2DP59 & Os07g0108500 & LOC_Os07g01780 & Chr7 & 457738 & 460218 & 2481 & 486 & 5.18 & 18121.8 \\
\hline OsC2DP60 & Os07g0165100 & LOC_Os07g07070 & Chr7 & 3485498 & 3489389 & 3892 & 3114 & 9.1 & 89297.46 \\
\hline OsC2DP61 & Os07g0260400 & LOC_Os07g15680 & Chr7 & 9105495 & 9112919 & 7425 & 2517 & 6.84 & 94957.94 \\
\hline OsC2DP62 & Os07g0409100 & LOC_Os07g22640 & Chr7 & 12747509 & 12760678 & 13170 & 1548 & 8.51 & 55377.37 \\
\hline OsC2DP63 & Os07g0483500 & LOC_Os07g30020 & Chr7 & 17687049 & 17692496 & 5448 & 3036 & 8.85 & 116382.9 \\
\hline OsC2DP64 & Os07g0500300 & LOC_Os07g31720 & Chr7 & 18840247 & 18842537 & 2291 & 522 & 6.51 & 18916.74 \\
\hline OsC2DP65 & Os07g0501700 & LOC_Os07g31830 & Chr7 & 18911787 & 18914891 & 3105 & 501 & 5.71 & 18739.53 \\
\hline OsC2DP66 & Os07g0585000 & LOC_Os07g39620 & Chr7 & 23746456 & 23748732 & 2277 & 1338 & 4.55 & 46052.94 \\
\hline OsC2DP67 & Os07g0670200 & LOC_Os07g47390 & Chr7 & 28339687 & 28340470 & 784 & 699 & 9.32 & 24564.25 \\
\hline OsC2DP68 & Os07g0670300 & LOC_Os07g47400 & Chr7 & 28344133 & 28345412 & 1280 & 933 & 9.43 & 32738.44 \\
\hline OsC2DP69 & Os07g0694000 & LOC_Os07g49330 & Chr7 & 29545825 & 29548981 & 3157 & 1797 & 6.15 & 67783.01 \\
\hline OsC2DP70 & Os08g0300200 & LOC_Os08g20544 & Chr8 & 12331526 & 12342183 & 10658 & 1269 & 5.29 & 61544.93 \\
\hline OsC2DP71 & Os08g0492400 & LOC_Os08g38440 & Chr8 & 24326030 & 24332435 & 6406 & 2850 & 5.37 & 105788.6 \\
\hline OsC2DP72 & Os08g0562600 & LOC_Os08g44850 & Chr8 & 28170358 & 28172330 & 1973 & 876 & 4.77 & 30344.58 \\
\hline OsC2DP73 & Os09g0251800 & LOC_Os09g07800 & Chr9 & 3949844 & 3953272 & 3429 & 516 & 5.6 & 19221.11 \\
\hline OsC2DP74 & Os09g0421300 & LOC_Os09g25390 & Chr9 & 15222652 & 15226444 & 3793 & 2454 & 6.02 & 89964.53 \\
\hline OsC2DP75 & Os09g0516900 & LOC_Os09g34130 & Chr9 & 20146860 & 20154260 & 7401 & 1839 & 5.88 & 67647.43 \\
\hline OsC2DP76 & Os09g0538800 & LOC_Os09g36770 & Chr9 & 21213298 & 21223349 & 10052 & 1620 & 6.33 & 61417.39 \\
\hline OsC2DP77 & Os09g0543100 & LOC_Os09g37100 & Chr9 & 21391774 & 21397949 & 6176 & 2565 & 6.51 & 95117.24 \\
\hline OsC2DP78 & Os09g0571200 & LOC_Os09g39770 & Chr9 & 22805610 & 22807049 & 1440 & 873 & 5.97 & 30468.78 \\
\hline OsC2DP79 & Os10g0524400 & LOC_Os10g38060 & Chr10 & 20377031 & 20386096 & 9066 & 3141 & 7.29 & 116544.8 \\
\hline OsC2DP80 & Os11g0183800 & LOC_Os11g08090 & Chr11 & 4233460 & 4243344 & 9885 & 6354 & 5.42 & 227943.6 \\
\hline OsC2DP81 & Os12g0187500 & LOC_Os12g08670 & Chr12 & 4397152 & 4400640 & 3489 & 2307 & 6.43 & 129412.6 \\
\hline OsC2DP82 & Os12g0562400 & LOC_Os12g37560 & Chr12 & 23048705 & 23053324 & 4620 & 1773 & 8.97 & 22230.7 \\
\hline
\end{tabular}

To further detect the diverse functions of OsC2DPs, polygenetic relationships and gene and domain structures were analyzed; the results are shown in Fig. 1 and Fig. 2. The NJ-Tree showed that 82 members were divided into seven groups, among which, most (19 members) gathered in Group-III and the least (3 
members) gathered in Group-IV (Fig. 1). In the structure analysis, the C2 domains were consistently identified, and single or multiple C2 domains were consistently present in each family member. The members of the seven groups had diverse structures, and the structure effectively supported the grouping results of the polygenetic tree (Fig. 2). In Group-I, 12 of the 13 members contained two Phospholipase D/Transphosphatidylase domains and a single Phospholipase D C-terminal domain. In particular, only OsC2DP12 showed other types and had distant relationships with other group members. Further, the results of multiple sequence alignment showed that this domain was highly conserved among the members (Additional file 2: Fig. S3). Group-II and Group-V showed similar domain structures (only a single C2 domain was found). However, most members of Group-II had a simple gene structure that contained one exon; Group-V members showed opposite results with multiple exon regions. Group-III had 19 members with one cluster of polygenetic tree, based on the mixture structure, we further divided them into three classes (A, B, and C); Class-C is a special class that showed a simple gene structure and a Phosphoribosyltransferase C-terminal. The results of multiple sequence alignment revealed a high homology between members of this class (Additional file 2: Fig. S4). In Group-VI and Group-VII, there displayed similar groupings divided into three classes (A, B, and C) according to the diverse domain structure. Further, in Group-VI Class-A and Class-C, there were single and two highly conserved domains, respectively. Synaptotagmin, SMP domain in Class-A (Additional file 2: Fig. S5); Phosphatidylinositol-specific phospholipase C, X domain and Phospholipase C, phosphatidylinositol-specific, Y domain in Class-C (Additional file 2: Fig. S6).

These results suggest that OsC2DPs as a widely gene family and performed diverse roles in plants; Members with a close relationship might possess similar functions; and diverse domains also involve differentiation in each group. Notably, the most conserved phospholipase-related domains were found in OsC2DPs, implying that they might be involved in abiotic stress, ion transporters, or exchange functions.

\section{Synteny analysis and $\mathrm{Ka} / \mathrm{Ks}$ ratio of OsC2DPs in rice and others genome}

The analysis of tandem or segmental duplications could explain the derivation of gene family duplication events. According to a previous study, alignment ratios greater than $70 \%$ were identified as duplications while gene pairs within the closed region (100 kb) were selected as tandem duplications. The results are shown in Fig. 3, red color and other color lines represent tandem and segmental duplications, respectively. Six tandem duplications (OsC2DP6/7, OsC2DP50/51, OsC2DP51/52, OsC2DP50/52, OsC2DP58/59, and OsC2DP67/68) and eight segmental duplications (OsC2DP11/46, OsC2DP2/43, OsC2DP10/67, OsC2DP20/36, OsC2DP13/54, OsC2DP14/53, OsC2DP30/69, and OsC2DP72/78) were found in the rice genome. Interestingly, three genes, OsC2DP50/OsC2DP51/OsC2DP52, involved a co-duplication event in a narrow region (19.784 kb), implying that these genes might have the same function or signaling pathway, or possess a redundancy effect in the regulation process. We also analyzed the duplication events of C2DPs between rice and other popular crop genomes. A total of 96, 100, 93, 82, 187, and 302 C2DPs were identified in Arabidopsis, Barley, Maize, Sorghum, Soybean, and Wheat genomes, respectively; the results are shown in Additional file 2: Fig. S7. Among these, Arabidopsis and wheat showed the most distant and close relationship with rice, with 8 and 176 duplications, respectively (Additional file 2: Fig. S7A and F). A total of 53, 83, 74, and 26 duplications were found between rice and barley, maize, sorghum, and soybean (Additional file 2: Fig. S7B-E). Such finding indicates that the OsC2DP gene family has expanded and evolved through genome duplication in rice or infusion in other crops.

In genetic studies, the Ka/Ks ratio represents the comparison of non-synonymous substitution rate (Ka) and the synonymous substitution rate (Ks) of duplications. Therefore, the $\mathrm{Ka} / \mathrm{Ks}$ ratio could determine whether there is selective pressure for this gene pair [30]. In previous results, we identified duplications in rice and other genomes; thus, we calculated these duplications Ka/Ks ratio for understanding the evolutionary model for OsC2DPs. All Ka/Ks ratios of the tandem and segmental duplications were less than 1 (Table 2), suggesting that these duplications involved purify (negative) selection. The duplication results of Arabidopsis, barley, maize, sorghum, soybean, and wheat were similar to those of intra rice, with Ka/Ks ratios less than 1 (Additional file 1: Table S3-S8), involved purify (negative) selection. Notably, there was one tandem duplication (OsC2DP58/59) with a Ka/Ks ratio of 0.905143 (Table 2), which is close to 1 as the neutral evolution. Another gene pair (OsC2DP67- TraesCS2A02G115700) had a Ka/Ks ratio of 1.0688 in the rice-wheat duplications (Additional file 1: Table S8), suggesting that these homologs involved positive selection. The average Ka/Ks ratio in tandem duplications ( 0.561$)$ was found to be greater than that in segmental duplication (0.263) and in the comparison of other genomes $(0.076,0.248,0.205,0.236,0.114,0.255)$, implying that tandem duplications of intra-rice were more likely to involve positive selection in the evolutionary process. 
Table 2. Synteny analysis for OsC2DP gene family intra rice and rice genome

Tandem Duplication

$\mathrm{Ka}$

Ks

\begin{tabular}{|c|c|c|c|c|c|c|c|c|c|c|c|}
\hline $\begin{array}{l}\text { Gene } \\
\text { Symbol }\end{array}$ & Gene ID & $\mathrm{Chr}$ & Start & End & $\begin{array}{l}\text { Gene } \\
\text { Symbol }\end{array}$ & Gene ID & Chr & Start & End & & \\
\hline OsC2DP6 & LOC_Os01g60340 & Chr1 & 34902303 & 34906955 & OsC2DP7 & LOC_Os01g60350 & Chr1 & 34908079 & 34908079 & 0.15904 & 0. \\
\hline OsC2DP50 & LOC_Os06g40170 & Chr6 & 23908918 & 23913797 & OsC2DP51 & LOC_Os06g40180 & Chr6 & 23921940 & 23921940 & 0.09049 & 0.1 \\
\hline OsC2DP51 & LOC_Os06g40180 & Chr6 & 23921940 & 23924921 & OsC2DP52 & LOC_Os06g40190 & Chr6 & 23928702 & 23928702 & 0.16077 & 0.4 \\
\hline OsC2DP50 & LOC_Os06g40170 & Chr6 & 23908918 & 23913797 & OsC2DP52 & LOC_Os06g40190 & Chr6 & 23928702 & 23928702 & 0.16616 & 0.4 \\
\hline OsC2DP58 & LOC_Os07g01770 & Chr7 & 452093 & 460151 & OsC2DP59 & LOC_Os07g01780 & Chr7 & 457738 & 457738 & 0.00405 & $0 . c$ \\
\hline OsC2DP67 & LOC_Os07g47390 & Chr7 & 28339687 & 28340470 & OsC2DP68 & LOC_Os07g47400 & Chr7 & 28344133 & 28344133 & 0.40704 & 0.6 \\
\hline
\end{tabular}

Segmental Duplication

Gene

Symbol

OsC2DP11 LOC_Os01g72230

OsC2DP2

LOC_Os01g07760

OsC2DP10

OsC2DP20 LOC_Os02g42710

OsC2DP13 LOC_Os02g10480

OsC2DP14 LOC_Os02g10630

OsC2DP30 LOC_Os03g18010

OsC2DP72
LOC_Os08g44850

\section{Chr Start

Start End

Chr1

Chr1

Chr1

Chr2

Chr2

Chr2

Chr3

Chr8
$41882488 \quad 41886410$

$3724314 \quad 3729284$

40973255

25684044

5505016

5586939

10025401

28170358

\section{2}

25687149

5508425

5593645

10027931

28172330

\section{Gene \\ Gene ID}

Symbol

OsC2DP46

OsC2DP43

OsC2DP67

OsC2DP36

OsC2DP54

OsC2DP53

OsC2DP69

OsC2DP78
LOC_Os05g31720

LOC_Os05g07880

LOC_Os07g47390

LOC_Os04g44870

LOC_Os06g40704

LOC_Os06g40570

LOC_Os07g49330

LOC_Os09g39770
Chr Start

Chr5

Chr5

18466963

4255763

Chr7

28339687

Chr4 26558810

Chr6

24264404

Chr6 24187249

Chr7 29545825

Chr9 22805610

$\mathrm{Ka}$

Ks

\section{Expression profile analysis of OsC2DPs in rice tissues}

The change in transcriptional level of genes could better explain the vitality of a gene in diverse tissues and growth stages. To determine the specific function of the gene at spatiotemporal variations, we analyzed the transcriptome data for the detected expression of OsC2DPs during different tissues and stages in rice. Diverse expression patterns were observed in the OsC2DPs (Fig. 4). For example, OsC2DP16/40/67/81/etc. showed higher expression in EN (1-3) and $M L$, suggesting that these genes function in later mature stages. OsC2DP29/33/43/74/4/59/30/etc showed the highest expression in HP, suggesting that these genes may perform major functions in HP, specially. Additionally, such as gene OsC2DP47/39/44 or OsC2DP37/11/80/54, had higher expression in BP (1-4) while OsC2DP51/41/50/64/etc had lower expression in EN (1-3) (Fig. 4); these diverse expression patterns indicate that some genes involved group cluster, might be performed functions by cooperated with cluster genes. Based on this conclusion, we further analyzed the expression profiles of tandem and segmental duplications. OsC2DP50/51/52 showed three genes as co-tandem duplications that contained similar expression variations across the entire growth stage in rice (Additional file 2: Fig. S8A). OsC2DP58/59 showed differences at an earlier stage but maintained consistent expression variations in HL to SP (Additional file 2: Fig. S8B). Further, there were no significant similarity in phenomena in OsC2DP67/68 (Additional file 2: Fig. S8C). In segmental duplications, there were two gene pairs; the two gene pairs showed completely similar and partially similar expression variations, respectively (Additional file 2 : Fig. S9). Among these, OsC2DP14/53 and OsC2DP72/78 showed similar expression variations across the whole growth stage (Additional file 2: Fig. S9D and H). OsC2DP11/46 was similar from the BP4 to EN stage (Additional file 2: Fig. S9A) while OsC2DP13/54 showed similar variations from GS to BLS2 and from HS to ML (Additional file 2: Fig. S9B).

Taken together, OsC2DPs were found to be widely expressed in whole tissues and growth stages. Further, a diverse expression pattern was observed in the gene cluster, suggesting that duplications were highly possible involved the same functions and regulation pathways in special tissues and stages by cooperation expression variation.

\section{Prediction and analysis of thecis-acting elements in promoter regions of OsC2DPs}

Generally, the gene response to treatments or other functions would be achieved by changing the activity of the treatment-related cis-acting elements [31]. In this study, we analyzed the abiotic stress and phytohormone related cis-acting elements in the promoter region of OsC2PDs as the validated functions of OsC2DP gene family were related to abiotic and phytohormone; the results are shown in Additional file 2: Fig. S10 and detailed information is in presented in Additional file 1: Table S9. A total of seven types of abiotic stress responsive elements and five types of phytohormone responsive elements were identified: the cold-responsive element, LTR (CCGAAA); drought responsive element, MBS (CAACTG); salt responsive element, GT1GMSCAM4 (GAAAAA); heat responsive element, CCAATBOX1 (CCAAT); diverse light responsive elements, ACE (GACACGTATG), G-box (TACGTG), GT1-motif (GGTTAA), etc.; circadian element,

Page $7 / 21$ 
CAAAGATATC; and wound responsive element, WUN-motif (AAATTTCCT). Phytohormone responsive elements, including ABA (ABRE: ACGTG/CACGTG), IAA (TGA-element: AACGAC and AuxRR-core: GGTCCAT), GA (TATC-box: TATCCCA and GARE-motif: TCTGTTG), SA (TCA-element: CCATCTTTTT), and MeJA (TGACG-motif: TGACG and CGTCA-motif: CGTCA) were found. After statistical analysis, a total of 923 light responsive elements and 390 MeJA responsive elements were found as the most abundant cis-acting elements in OsC2DPs. For each gene, the light-responsive element most enriched for OsC2DP5 (28), OsC2DP29 (22), OsC2DP3 (20), and OsC2DP64 (20), implied that the functions of these gene functions were possible involved in the light signaling pathway in rice. In addition, some genes contained most elements with multiple functions, such as OsC2DP5 contained most elements related to salt and light; OsC2DP29 contained most elements related to cold, salt, and light; and OsC2DP28 contained most elements related to drought and heat. These results indicate that the OsC2DP gene family is widely involved in abiotic and phytohormone stress in rice.

\section{Expression analysis of OsC2DPs involved abiotic and phytohormone stress}

To further verify the function of genes in abiotic and phytohormone stress, we collected multiple RNA-seq data and performed qRT-PCR to detect the variation in transcriptional levels in OsC2DPs. First, the results of transcript profile revealed that under treatments of ABA, GA, IAA, cytokinin, SA, and JA, the family members exhibited diverse responses to phytohormones (Additional file 2: Fig. S11). For example, OsC2DP6, OsC2DP9, etc., these genes were consistently repressed by ABA, GA, IAA, and cytokinin; OsC2DP15, OsC2DP73, etc., these genes were consistently induced by these phytohormone; and OsC2DP16, OsC2DP15, etc., these genes had opposite expression changes under the treatments of SA and JA. In addition, among the three co-tandem duplications, OsC2DP50/51/52 showed a consistent response to SA and JA, and only OsC2DP50/51 remained consistent under treatments of ABA, GA, IAA, and cytokinin. These results suggest that OsC2DPs are divided into diverse patterns that are widely involved in the response to phytohormone stress; among these, the duplications might maintain similar functions or involve the same pathway for different stresses.

Subsequently, some abiotic-response genes were selected via previous cis-acting element analysis based on the major count of abiotic-related motifs. We performed qRT-PCR to verify the expression change of candidate abiotic-response genes; the results are shown in Fig. 5 and Additional file 2: Fig. S12. OsC2DP8/29/17 expression was significantly induced by cold stress, OsC2DP46/49/71 expression was repressed, and only OsC2DP19 showed no obvious change (Fig. 5A). Under heat shock stress, OsC2DP71/28/41 expression was induced and OsC2DP19/79 was first repressed but subsequently recovered (Fig. $5 B)$. Such findings suggest that OsC2DP71 expression was induced by cold and repressed by heat, ultimately indicating that this gene as a temperaturesensitive factor. In addition, OsC2DP8/9/28 expression was induced and OsC2DP25 was repressed by drought stress (Fig. 5C). Under salt and alkaline stress, OsC2DP29/5 expression was similarly induced by treatments, and OsC2DP46/49/41 showed diverse variation in response to these two stresses (Fig. 5D and E). In plants, light response is a key signal for plant growth and both photosynthesis and circadian rhythms affect plant growth and development [32]. Thus, we performed qRT-PCR under light and dark conditions. All candidate gene expression levels showed significant variation in different treatments (Additional file 2: Fig. S12). OsC2DP29/46 expression was induced and OsC2DP5 was repressed under dark conditions compared to that under light conditions (Additional file 2: Fig. S12A, B and C). For OsC2DP79/3/64, circadian rhythms were evidently observed under normal (light) growth condition (Additional file 2: Fig. S12D, E and F). In contrast, under dark conditions, the circadian rhythms of OsC2DP79/3 were disrupted, resulting in levelling out for expression (Additional file 2: Fig. S12D and E). OsC2DP64 expression showed an opposite trend compared to that found under the normal (light) condition (Additional file 2: Fig. S12F). These results suggest that these OsC2DPs widely involved diverse abiotic stresses; OsC2DP5/29/49/71 could respond to multiple stress treatments, possibly playing positive or negative roles in these functions.

\section{Bioinformatics predictions and enrichment of OsC2DPs}

In the last decade, miRNAs have been found in diverse plant species, influence plant growth development and change the survivability to biotic and abiotic stress [33], which are directly involved in the functions of miRNAs by cleavage or translation at the transcriptional level [34]. In this study, we analyzed the OsmiRNAs-OsC2DPs pathway and predicted the putative regulatory network using the miRNA database. A total of 167 unique potential OsmiRNAs-targets of OsC2DPs were identified, with miRNAs 19-24 nucleotides long (Additional file 1: Table S10), among these, functions of 146 unique targets as the cleavage and 21 unique targets as the translation were identified, suggesting that cleavage is the major function in the regulation of OsmiRNAs-targets of OsC2DPs. There were 28 OsC2DPs involved in one relevant network (Fig. 6) while the remaining 29 OsC2DPs were involved in fragmented networks (Additional file 2: Fig. S13). Among these, OsC2DP71 possessed 11 potential targeted OsmiRNAs; genes OsC2DP53/79/27/39/3 possessed 9/7/7/7/6 potential targeted OsmiRNAs, respectively; and OsmiR2927, OsmiR5809, OsmiR5075, and OsmiR5833 contained six, five, six, and six potential targets, as the most and major OsmiRNAs-target in the OsC2DP gene family. In addition, subfamily specific targets were identified: Group-I members, OsC2DP27/79/61 co-targeted by OsmiR5830; and Group-III members, OsC2DP32/34/47/21 co-targeted by OsmiR5833. As expected, OsC2DP50/51/52 was co-targeted by OsmiR2927 as the co-tandem duplications, jointly targeted with Group-I members, OsC2DP31/43/12 (Fig. 6). Additionally, we performed GO and KEGG enrichments analyses of the OsC2DP gene family. Results showed major functions involved membrane, plasma membrane, multiple metabolic process, phospholipase activity and ion binding of GO functions (Additional file 2: Fig. S14A). KEGG results showed that the major pathways were membrane and multiple metabolic processes (Additional file 2: Fig. S14B). Together, these results imply that OsC2DPs perform major functions in the cell membrane, possibly via ion combination and transportation.

\section{SNP genotyping and haplotype analysis of OsC2DPs}

In rice, Japonica and Indica, the major subspecies, contain different traits caused by many genotype variations; thus, we analyzed the SNP genotyping and abiotic-related haplotypes of OsC2DPs. First, we extracted all SNPs of the OsC2DPs promoter, UTR, exon, and intron regions from a 1.85 million high-quality core collection re-sequence data. A total of 2,861 SNPs were selected based on diverse gene positions. SNP annotation results showed that 424 SNPs were located in promoters; 219 SNPs were located in UTR regions; and 223 SNPs were located in exon regions, 124 of which were synonymous and 99 were nonsynonymous variants (Table 3). After removing the Adm variety, a PCA was carried out using OsC2DPs SNP data (only remained Jap and Ind varieties). 
Results showed that PC1 explained $87.55 \%$ and PC2 explained $12.55 \%$ of the variation, and two subspecies varieties were separated into two groups (Additional file 2: Fig. S14C).

Table 3. Summary for SNPs in OsC2DPs

\begin{tabular}{lllll}
\hline Variation type & Count & Variation in Exon & Count \\
\hline Promoter variation & 424 & & \\
\hline Upstream variation & 575 & & \\
\hline Downstream variation & 73 & & \\
\hline Upstream \& Downstream variation & 265 & & \\
\hline 5'UTR variation & 47 & & 124 \\
\hline 3'UTR variation & 172 & & 99 \\
\hline Intergenic variation & 178 & & \\
\hline Exonic variation & 223 & Synonymous variant & \\
\cline { 2 - 5 } & & Non-synonymous variant & 9 \\
\hline Intronic variation & 829 & & \\
\hline ncRNA_exonic variation & 75 & & \\
\hline
\end{tabular}

In the haplotype analysis, a set of cold-related phenotypes was associated with genotype data; this analysis was performed to identify the functional phenotype-related genotypes in different varieties. According to previous qRT-PCR results, OsC2DP17, OsC2DP29, and OsC2DP49 were selected as candidate genes for cold stress. For OsC2DP17, after filtering genotype data that SNPs contained missing or heterozygotes, eight SNPs were found in the intron, exon, and promoter regions (Fig. 7A). These SNPs formed four haplotypes for OsC2DP17 and the major varieties involved Hap1 and Hap4 (Fig. 7B), LD analysis results showed a strong LD relationship between each SNP pair (Fig. 7C). Haplotype network and variation analysis showed that there were two major groups, Hap1 and Hap2, which contained major Ind and Aus subspecies; Hap3 and Hap4 contained major Tej and Trj subspecies. Large genotype variations were also observed between these two groups (Fig. 7D). The association of phenotype-haplotype was analyzed, and a set of CT score (1-9 score) was used as the evaluation index. As shown in Fig. 7E and F, Hap1 and Hap2 showed significantly cold sensitivity (higher CT score) compared with Hap3 and Hap4, respectively, indicating that Hap1 and Hap2 conferred major sensitivities to cold tolerance in OsC2DP17. By comparing these SNPs, we found a key SNP, SNP 8 , located at promoter $-1652 \mathrm{bp}$, which made a nucleotide change from $\mathrm{T}$ to $\mathrm{C}$ (named as $-1652^{\top}$ and $-1652^{\mathrm{C}}$ ). The SNP 8-haplotype analysis result showed that $-1652^{\top}$ conferred major tolerance to cold stress compared with $-1652^{C}(P=2.492 E-11)$. Interestingly, through promoter analysis, we found that SNP 8 was located in the cold-related motif, MYCCONSENSUSAT (CANNTG) (Fig. 7G) [35], and its mutant possibly led to a change in gene expression under cold stress.

For OsC2DP29 and OsC2DP49, six SNPs were selected in both genes by removing missing or heterozygote data, which were found in the intron and exon regions of OsC2DP29 and the promoter, intron, and exon regions of OsC2DP49, respectively (Additional file 2: Fig. S15A and B, Additional file 2: Fig. S16A and B). LD analysis of both genes revealed similar results to OsC2DP17, which had a strong LD relationship between each SNP pair (Additional file 2: Fig. S15C and Additional file 2: Fig. S16C). The haplotype network showed that the haplotypes of OsC2DP29 were divided into two groups: Hap1, Hap4, and Hap5, which contained the most Ind, Aus, and Adm varieties (Additional file 2: Fig. S15D); and Hap2 and Hap3, which included most Trj and Tej varieties. Such finding suggests that OsC2DP29 is a Jap-Ind specific genotyping gene. As expected, the phenotype-associated results showed that Hap3 and Hap2 had significantly lower CT scores than other Haps (Additional file 2: Fig. S15E), suggesting that the genotypes of Trj and Tej conferred stronger cold tolerance in this population, which also aligns with the general difference in temperature sensitivity between Indica and Japonica subspecies. Similar to the above analysis, through a comparison of each SNP, SNP3 caused a nucleotide change from A to T (named as $1894^{\mathrm{A}}$ and $1894^{\top}$ ) located at 1894 bp of intron region was selected, due to this SNP genotypes of Hap2 and Hap3 resulted in a phenotypic difference. Thus, by analysing independent SNP haplotypes, $1894^{\mathrm{A}}$ was found to show highly significant variations in CT score compared with $1894^{\top}$ (Additional file 2: Fig. S15F), suggesting that this SNP may be a key and functional SNP for the genotyping of OsC2DP29 in cold tolerance. Similarly, five haplotypes of OsC2DP49 were roughly divided into Jap (Hap1, Hap2, and Hap3) and Ind (Hap4 and Hap5) groups (Additional file 2: Fig. S16D), only Hap3 and Hap4 contained mixture varieties. The phenotype-haplotype associated results showed that Hap1 and Hap2 had significantly lower CT score in populations (Additional file 2: Fig. S16E). A key SNP, SNP3, changed the nucleotide G to A (named as -316 ${ }^{G}$ and $-316^{A}$ ), which was located at -316 bp of the promoter region. The SNP-haplotype showed that - 316A had a significantly lower CT score for OsC2DP49 (Additional file 2: Fig. S16F), similar to that of $1894^{\mathrm{A}}$ in OsC2DP29.

These results suggest that OsC2DP17, OsC2DP29, and OsC2DP49 are highly possible involved in cold stress, diverse haplotypes were identified, which supported the potential theoretical foundation of the relationship between genotype variations and stress tolerance for present population in rice.

\section{Discussion}

\section{Identification and polygenetic relationship of OsC2DPs in rice genome}


Recently, gene family studies have been constantly reported. An extensive study of gene family analysis could support a comprehensive understanding of the biological functions of each family. For example, OsANTH3, a member of the AP180 N-terminal homology domain-containing protein, has been reported to be involved in pollen-related functions in rice [36]. Further, the MAPKKK gene family is involved in drought stress in cotton [37], which supports the wide prediction of gene function and theoretical foundation for homologs of evolutionary relationships. The C2 domain contain proteins, which show at least one $\mathrm{C} 2$ domain that acts as a lipid binding domain and a major sensor of diverse $\mathrm{Ca}^{2+}$-mediated cellular processes [14]. Most C2DPs can perform functions and cooperate with other transmembrane domains, such as phospholipase domain and synaptotagmin [38], and maintain highly conserved characteristics in the evolutionary process. In the present study, we used a conserved HMM model, performed blast function in the rice protein database, and identified 82 OsC2DPs at each chromosome (Additional file 1: Table S1, Additional file 2: Fig. S1). Few gene clusters were also found in chromosomes 1, 6, and 7. A previous study showed that gene clusters produced tandem duplication events in the genome, implying that OsC2DPs might have duplication events in cluster regions. The prediction of subcellular localization showed OsC2DPs protein play a role throughout the cell (Additional file 1: Table S2), suggesting that involved multiple regulation network of growth development process. Interestingly, major proteins were located in the cytoplasm and nucleus (Additional file 2: Fig. S6); thus, we speculated that some proteins played roles as regulation transporters for shuttling between the nucleus and membrane. A similar function was also reported in a previous study, including the localization of the protein, OsRAN2, in the nucleus and cytoplasm, which could regulate the transport of proteins and RNA across the nuclear envelope and play a role in mitotic spindle and nuclear envelope (NE) assembly, overexpression of OsRAN2 could enhanced tolerance to salt stress in rice [39]. Another study reported that the protein, OsSAP1, could interact with the proteins, OsSAP11 and OsRLCK253, and that this interaction occurred in karyotheca, plasmalemma, and endonuclear, and these proteins also perform roles in drought and salt tolerance in plants [40]. Suggested that some members of OsC2DPs might display similar functions for nuclear-cytoplasmic shuttling, perform interaction function, and act in abiotic stress in rice. Polygenetic analysis revealed that a total of seven groups were divided into 82 family members according to domain and gene structure, and multiple transmembrane region domains were found in the different groups (Fig. 2). A previous study also reported similar polygenetic results for C2DPs; in Arabidopsis, the multiple $\mathrm{C} 2$ domain and transmembrane region proteins were reported [27], the members of rice homologs in a previous study were similar to members of group-III Class-C in the present study; and in cotton, rice homologs of MCTPs were found in a polygenetic study that is consistent with the present study [28]. In rice, previous study identified OsNTMC2 members were identified [29], OsNTMC2s contain a highly conserved N-terminal TM domain, SMP domain, and C-terminal C2 domain, which is consistent with Group-VI Class-A in the present study, validating the conservatism of each group and the accuracy of our analysis (Fig. 2).

\section{Duplications events performed in OsC2DPs}

Normally, duplications contain conserved domains or sequences that may involve similar functions in plants [41]. Among these, tandem duplications as major and commonly evaluated mechanisms for gene family expansion which produced novel genes and clusters into gene families, had an impact on a small number of genes, but had a significant contribution to gene family expansion [42]. In the present study, a total of $8,53,83,74,26$, and 176 duplications were identified between rice and Arabidopsis, barley, maize, sorghum, soybean, and wheat (Additional file 2: Fig. S7, Tables S3-S8), implying the occurrence of duplication events and the potential genome expansion of OsC2DPs from other species, especially the wheat genome. In rice, a total of six tandem and eight segmental duplications were found (Fig. 3, Table 2), suggesting that these duplications might involve similar functions in rice; this hypothesis was also validated through further analysis. In the expression file data, the tandem duplications, OsC2DP50/51/52 and OsC2DP58/59, showed a similar expression variation tendency with rice growth stages. Evidently, co-duplications OsC2DP50/51/52 expression showed the same response to diverse phytohormones and involved the same potential regulation pathway as the miRNA-targets of miR2927 (Fig. 6), providing exact evidence for the predicted functions of coduplications OsC2DP50/51/52. A previous study reported that tandem duplication events occurred more frequently than other events and formed large gene copies and allelic variations [43]. Tandem duplications are widely involved in the control of stress tolerance and membrane functions in rice and Arabidopsis [44, 45], or the transduction of the signaling pathways in legumes [46].

\section{Elements prediction and expression analysis of OsC2DPs}

Based on the prediction of cis-acting elements in the promoter regions of OsC2PDs, many phytohormone- and abiotic stress-related motifs were found (Additional file 2: Fig. S10), implying that OsC2DPs may be involved in phytohormones and abiotic stress. Furthermore, expression profile data of phytohormone treatments validated the previous hypothesis, and qRT-PCR was performed to identify expression variations under abiotic stress treatments in some putative functional genes, which suggested that OsC2DPs are involved in phytohormones and abiotic stress. In a previous study, some OsC2DPs functions were reported in transgenic plants, such as OsPBP1(LOC_Os04g44870) was named as OsC2DP36, involved Ca2 + concentration-dependent phospholipid-binding activity and localized in the cytoplasm and nucleus, whereas shuttling at the plasma membrane by increased intracellular Ca ${ }^{2+}$ [47]. The rice no pollen gene (LOC_Os06g40570) was named OsC2DP53 and is responsible for the production and development of pollen, which might be related to $\mathrm{Ca}^{2+}$ and phosphoinositol signaling pathways depending on the C2 and GRAM domains [48]. OsC2DP (LOC_Os09g39770) was named OsC2DP78, a functional mutant that changed tolerance to salt, showed variations in $\mathrm{Na}^{+}$concentration and $\mathrm{K}^{+} / \mathrm{Na}^{+}$ratio [26]. Thus, except for members of OsNTMC2s and MCTPs included in previous studies, other identified OsC2DP family members that involved present study also widely performed diverse functions of growth development in rice.

\section{Identification of potential variation alleles for future breeding}

SNP genotyping was performed PCA analysis using the SNP data of OsC2DPs (Additional file 2: Fig. S14C). The significant Japonica-Indica polarization of OsC2DPs supports a potential future research direction. Further, diverse genotypes of allelic variation could produce different tolerances to abiotic stress in multiple subspecies [49,50]. Haplotype analysis revealed that OsC2DP17, OsC2DP29, and OsC2DP49 possessed diverse alleles among the core collection population, which generated different cold tolerance for each variety (Fig. 7, Additional file 2: Fig. S15 and Additional file 2: Fig. S16). Interestingly, we identified key SNPs that significantly impacted tolerance in these candidate genes. The SNPs $-1652^{\top}, 1894^{\mathrm{A}}$, and $-316^{\mathrm{A}}$ were located in intron and promoter regions, we speculated that these SNPs might possess strong LD levels coordinated with crucial translation or functional domain region of each gene, thereby 
producing a diverse phenotype in rice. In summary, these haplotypes and SNP-haplotypes are supported potential opportunities for enhanced related tolerance in future molecular design breeding.

\section{Conclusions}

In summary, we are provided first study to comprehensively characterize the C2DP gene family in rice. Herein, 82 members and multiple transmembrane domains were identified by phylogenetic analysis. The roles of the OSC2DP gene in abiotic stress were identified by bioinformatics prediction, transcript profile analysis, and qRT-PCR experiments. Further, the polarization genotypes between diverse rice subspecies and putative functional haplotypes were characterized by SNP genotyping and haplotype analysis. The findings of this study contribute to a better understanding of the functions of the $\mathrm{C} 2$ domain and future functional characterization of OsC2DPs.

\section{Methods}

\section{Identification, chromosome distribution, and localization of OsC2DPs}

In the present study, rice genome and protein sequences were downloaded from the Phytozome database (http://www.phytozome.net). To identify the OsC2DP gene family members in rice, a Hidden Markov model (PF00168) of the C2-domain was downloaded from the PFAM database that as trigged for blast in rice Proteomic sequence, with E-value threshold less than $1 \mathrm{e}^{-5}$ [51]. The filtered blast hits were submitted to the InterPro (https://www.ebi.ac.uk/interpro/) and SMART (http://smart.embl-heidelberg.de/) databases and were retrieved for the C2-domain. The diverse lengths of OsC2DPs were calculated using TBtools [52], and the physical properties ( $\mathrm{pl}$ and Mw) were analyzed using the ExPASy website

(https://web.expasy.org/compute_pi/). According to the prepared gene positions obtained from the rice genome file, chromosome distribution and visualization were performed using the MapGene2Chromosome V2 (http://mg2c.iask.in/mg2c_v2.0/) according to the official default procedure. To predict the subcellular localization of OsC2DPs, full-length proteins of OsC2DPs were submitted to the website tool, CELLO v.2.5: subCELlular LOcalization predictor [53], and the final localization results were determined by the major score of each position in every family member.

\section{Polygenetic, structure and multiple sequence alignment analyses}

To perform the polygenetic relationship of OsC2DPs, the full-length protein of OsC2DPs leaded into MEGA software [54]. Further, a polygenetic tree was constructed using NJ-tree functions with 1000-times bootstrap iterations, and a final plot of visualization was generated using tools on the iTOLs website (https://itol.embl.de/) [55]. To identify multiple structure of OsC2DPs, full-length protein sequences were submitted and analyzed using the PFAM database. Visualization was performed using each domain length and position in the website Gene Structure Display Server (http://gsds.gao-lab.org/) [56]. Gene structures were analyzed using the software, TBtools, and the rice gff3 file. For multiple sequence alignment, whole protein sequences of OsC2DPs were aligned using ClustalW 2.0 [57] software and visualized using the website, Sequence Manipulation Suite (http://www.bioinformatics.org/) [58], where a consistent color represents a highly conserved amino acid.

\section{Synteny and $\mathrm{Ka} / \mathrm{Ks}$ analysis}

To analyzed synteny in OsC2DPs, C2DPs in the other six plant species were identified using the same procedure that used in the identification of OsC2DPs. BLAST calculations were performed between the genome of rice and those of other species using the BLAST software [59]. Thereafter, multiple genome synteny analysis was performed using MCScanX software [60]. Among these, duplications were determined by an alignment ratio over 70\% and blast ratio over $70 \%$ while tandem duplications were determined based on the involvement within a $100 \mathrm{~kb}$ region [61]. All duplications of C2DPs were filtered from whole genome duplications, and plots were visualized using Circos software using default parameters [62]. For Ka/Ks calculation, full-length protein sequences of all duplication C2DPs were aligned using ClustalW 2.0. Thereafter, Ka, Ks, and Ka/Ks ratios were calculated using the software, KaKs_Calculator 2.0, following the default procedure [63].

\section{Expression profile analysis and prediction of cis-acting elements}

For collected expression profile data, multiple RNA-seq analysis results were selected from the NCBI GEO database. The rice tissue-specific expression and duplication expression pattern data followed GSE19024 [64], treatments of ABA, GA, IAA, cytokinin, SA, and JA data followed GSE39429 and GSE37557 [65, 66]. After the OsC2DP expression data were filtered from these data profiles, the mean value and related fold change were calculated by comparison to the control (no treatment). Visualization of the heatmap was performed using the R program. Data were analyzed using log, row normalization, and cluster analysis. For prediction of cis-acting elements for OsC2DPs, all promoters of OsC2DPs were extracted using the software, TBtools, and the promoter was determined using $2000 \mathrm{bp}$ upstream sequences of the transcription initiation site for each family member. Element prediction was performed using the database, PlantCARE [67], and the related motifs were classified according to database annotations.

\section{Plant growth, treatment, and quantitative real-time PCR analysis}

To validate the OsC2DP expression response to abiotic stress, common variety Nipponbare (IT003166) was selected for experiment, that was obtained from Rural Development Administration (RDA) National Institute of Agricultural Sciences (http://genebank.rda.go.kr/initMain.do). Plants were grown to 2 leaves stage seedlings using Yoshida growth solutions under normal growth conditions $\left(28^{\circ} \mathrm{C} / 26^{\circ} \mathrm{C}, 12 \mathrm{~h} / 12 \mathrm{~h}\right)$. Thereafter, they were collected under treatments of cold $\left(4^{\circ} \mathrm{C}\right)$, heat $\left(42^{\circ} \mathrm{C}\right)$, drought $(\mathrm{dry})$, salt $(200 \mathrm{mM})$, and alkaline $\left(0.15 \% \mathrm{Na}_{2} \mathrm{CO}_{3}\right)$ stress. For light response, Nipponbare plants were grown to 2 leaves stage and leaf samples were collected under dark and normal (light) conditions. The time points for collection were $0 \mathrm{~h}, 1 \mathrm{~h}$, and $3 \mathrm{~h}$ for cold, heat shock, drought, salt, and alkaline stress; and $0 \mathrm{~h}, 12 \mathrm{~h}, 24 \mathrm{~h}, 36 \mathrm{~h}$, and $48 \mathrm{~h}$ for light and dark treatments. Total RNA was extracted from the collected samples using the 
RNeasy Plant Mini Kit (QIAGEN). All samples were treated with RNase-Free DNase (QIAGEN) to remove genomic DNA. Complementary DNA was synthesized using the SuperScript III Kit (Thermo). For primer design, each gene cds sequence was inputted and produced by the software, Primer5. The gene, Actin, served as the internal control to ensure corrected accuracy of results; the total list is shown in Additional file 1: Table S11. qRT-PCR was performed using SYBR Green PCR Kit (QIAGEN) and Rotor-Gene Q (QIAGEN) system, following the default production procedure. Each reaction was repeated three times; the mean value was calculated and the $-\triangle \triangle C T$ method was used to calculate the relative expression [68].

\section{miRNA target prediction and enrichment analysis of OsC2DPs}

To predict the potential miRNA targets of OsC2DPs, the full-length of the cds sequences of OsC2DPs was extracted and predicted using the miRNA database, psRNATarget [69]. The expectation threshold was set to 4 for filtered pseudo results, and visualization was performed using the Cytoscape software [70]. For gene ontology and KEGG enrichment, the PlantGSEA database (http://bioinformatics.cau.edu.cn/PlantGSEA/) was employed for OsC2DPs [71]. The results were filtered by adjust $\mathrm{p}$-value less than 0.05 , showing 14 major enrichment functional terms, and visualization was performed using the $\mathrm{R}$ program.

\section{SNP genotyping and haplotype analysis}

A core collection set of rice was employed in the present study. A total of 137 varieties containing multiple Jap, Ind, and Adm were collected from 28 countries [72]. Approximately 1.85 million high-quality SNPs were filtered from raw re-sequencing data, following a previous study [73]. For SNP genotyping of OsC2DPs, all SNPs located at the promoter, UTR, intron, and exon of OsC2DPs formed a 2861 SNP set, the annotations were performed using website tools http://bioinfo.sibs.ac.cn/. PCA analysis and visualization were performed using R program by default functions using 2861 SNPs data. For haplotype analysis, SNPs located at OsC2DP17, OsC2DP29, and OsC2DP49 were collected following the above descriptions and the missing and heterozygote data were filtered. Phenotype data included an evaluation of the CT score following a previous study [74]. Haplotype grouping was carried out based on the SNP variations between each Hap. Significant tests of phenotype-haplotype and SNP-haplotype were carried out using SPSS software; different letters represent differences. Visualization of the haplotype network was performed using the software, PopArt [75]. LD analysis was performed using Haploview software [76].

\section{Abbreviations}

C2DP: C2 domain contain protein

MCTP: multiple C2 domains and transmembrane region protein

NTMC2: N-terminal-TM-C2 domain proteins

Ca: Calcium

miRNA: microRNA

MW: Molecular weight

NJ: Neighbor-joining

pl: Isoelectric point

HMM: Hidden Markov Model

Ka: Non-synonymous substitution rate

Ks: Synonymous substitution rate

ABA: Abscisic acid

IAA: Auxin

GA: Gibberellin

SA: Salicylic acid

MeJA: Methyl jasmonate

SNP: Single nucleotide polymorphism

Hap: Haplotype

LD: Linkage disequilibrium

CT: Cold tolerance

Trj: Tropical japonica

Tej: Temperate japonica 
Ind: Indica

Adm: Admixture

qRT-PCR: Quantitative real-time polymerase chain reaction

\section{Declarations}

\section{Ethics approval and consent to participate}

Not applicable.

\section{Consent for publication}

Not applicable.

\section{Availability of data and materials}

The genome, protein, and Generic Feature Format files of rice, Arabidopsis thaliana, Barley, Maize, Sorghum, Soybean, and Wheat were downloaded from the Phytozome database (http://www.phytozome.net) and Ensemble FTP (http://plants.ensembl.org/info/data/ftp/index.html). The RNA-seq data generated during the current study have been deposited and publicly available at the National Center of Biotechnology Information repository, GEO database (https://www.ncbi.nlm.nih.gov/geo/), accession numbers: GSE19024, GSE39429 and GSE37557. All datasets generated and analyzed during this study are included in this article and its additional files.

\section{Competing interests}

The authors declare that they have no competing interests.

\section{Funding}

This work was supported by grants from the Basic Science Research Program through the National Research Foundation of Korea (NRF) funded by the Ministry of Education, Science and Technology (NRF-2021R1A4A2001968) provided by STK. The funders have no role in the design of the study and collection, analysis, and interpretation of data and in writing the manuscript.

\section{Authors' contributions}

$\mathrm{HZ}$ and SWK conceived and designed research. $\mathrm{HZ}$ conducted experiments, $\mathrm{HZ}$ and $\mathrm{YZ}$ collected and analyzed the data, $\mathrm{HZ}$ wrote the preliminary manuscript. JS, YJK and STK gave critical revision of the manuscript. All authors have read and approved the manuscript.

\section{Author information}

Affiliations

Department of Plant Bioscience, College of Natural Resources and Life Science, Pusan National University, Miryang 50463, Republic of Korea

Hongjia Zhang, Jeonghwan Seo, Sun Tae Kim, Soon-Wook Kwon

College of Agricultural, Yangtze university, Jingzhou, 434000, China

Yuting Zeng

Department of Life Science \& Environmental Biochemistry, College of Natural Resources and Life Science, Pusan National University, Miryang 50463, Republic of Korea

Yu-Jin Kim

Corresponding authors

Correspondence to Soon-Wook Kwon

\section{Acknowledgements}

Not applicable.

\section{Additional information}

Publisher's Note

Springer Nature remains neutral with regard to jurisdictional claims in published maps and institutional affiliations. 


\section{References}

1. Kirkby E, Pilbeam D. Calcium as a plant nutrient. Plant, Cell Environ. 1984,7(6):397-405.

2. Du L, Ali GS, Simons KA, Hou J, Yang T, Reddy A, Poovaiah B. Ca 2+/calmodulin regulates salicylic-acid-mediated plant immunity. Nature. 2009,457(7233):1154-1158.

3. Kudla J, Batistič O, Hashimoto K. Calcium signals: the lead currency of plant information processing. The Plant Cell. 2010,22(3):541-563.

4. Sanders D, Brownlee C, Harper JF. Communicating with calcium. The Plant Cell. 1999,11(4):691-706.

5. Xiong L, Schumaker KS, Zhu J-K. Cell signaling during cold, drought, and salt stress. The plant cell. 2002,14(suppl 1):S165-S183.

6. Luan S, Kudla J, Rodriguez-Concepcion M, Yalovsky S, Gruissem W. Calmodulins and calcineurin B-like proteins: Calcium sensors for specific signal response coupling in plants. The Plant Cell. 2002,14(suppl 1):S389-S400.

7. Plant PJ, Yeger H, Staub O, Howard P, Rotin D. The C2 domain of the ubiquitin protein ligase Nedd4 mediates Ca2+-dependent plasma membrane localization. J Biol Chem 1997, 272(51):32329-36.

8. Farah CA, Sossin WS. The role of C2 domains in PKC signaling. Calcium Signaling. 2012:663-683.

9. Kopka J, Pical C, Hetherington AM, Müller-Röber B. Ca 2+/phospholipid-binding (C 2) domain in multiple plant proteins: novel components of the calciumsensing apparatus. Plant Mol Biol. 1998,36(5):627-637.

10. Merithew E, Lambright DG. Calculating the potential of C2 domains for membrane binding. Dev Cell. 2002,2(2):132-133.

11. Schapire AL, Voigt B, Jasik J, Rosado A, Lopez-Cobollo R, Menzel D, Salinas J, Mancuso S, Valpuesta V, Baluska F. Arabidopsis synaptotagmin 1 is required for the maintenance of plasma membrane integrity and cell viability. The Plant Cell. 2008,20(12):3374-3388.

12. Molz L, Chen Y-W, Hirano M, Williams LT. Cpk is a novel class of Drosophila Ptdlns 3-kinase containing a C2 domain. J Biol Chem. 1996,271(23):1389213899.

13. Maeda I, Kohara Y, Yamamoto M, Sugimoto A. Large-scale analysis of gene function in Caenorhabditis elegans by high-throughput RNAi. Curr Biol. 2001,11(3):171-176.

14. Cho W, Stahelin RV. Membrane binding and subcellular targeting of C2 domains. Biochimica et Biophysica Acta (BBA)-Molecular and Cell Biology of Lipids. 2006,1761(8):838-849.

15. Nalefski EA, Falke JJ. The C2 domain calcium-binding motif: structural and functional diversity. Protein Sci. 1996,5(12):2375-2390.

16. Zhai X, Gao Y-G, Boldyrev IA, Malinina L, Patel DJ, Molotkovsky JG, Chalfant CA, Brown RE. Regulation of Membrane Binding by the C2-Domain of Cytoplasmic Phospholipase A2 by Ceramide-1-Phosphate and Calcium. Biophys J. 2017,112(3):392a-393a.

17. Zhang X, Jiang S, Mitok KA, Li L, Attie AD, Martin TF. BAIAP3, a C2 domain-containing Munc13 protein, controls the fate of dense-core vesicles in neuroendocrine cells. J Cell Biol. 2017,216(7):2151-2166.

18. Souroujon MC, Mochly-Rosen D. Peptide modulators of protein-protein interactions in intracellular signaling. Nat Biotechnol. 1998,16(10):919-924.

19. Brandman R, Disatnik M-H, Churchill E, Mochly-Rosen D. Peptides derived from the C2 domain of protein kinase C epsilon (epsilon PKC) modulate epsilon PKC activity and identify potential protein-protein interaction surfaces. The Journal of biological chemistry. 2006,282(6):4113-4123.

20. Rahier R, Noiriel A, Abousalham A. Functional characterization of the N-Terminal C2 domain from Arabidopsis thaliana phospholipase Da and D 3 . BioMed research international. 2016,2016.

21. Premkumar A, Lindberg S, Lager I, Rasmussen U, Schulz A. Arabidopsis PLDs with C2-domain function distinctively in hypoxia. Physiol Plant. 2019,167(1):90-110.

22. Cheung M-Y, Ngo JC-K, Chen Z, Jia Q, Li T, Gou Y, Wang Y, Lam H-M. A structure model explaining the binding between a ubiquitous unconventional Gprotein (OsYchF1) and a plant-specific C2-domain protein (OsGAP1) from rice. Biochem J. 2020,477(20):3935-3949.

23. Vaddepalli P, Herrmann A, Fulton L, Oelschner M, Hillmer S, Stratil TF, Fastner A, Hammes UZ, Ott T, Robinson DG. The C2-domain protein QUIRKY and the receptor-like kinase STRUBBELIG localize to plasmodesmata and mediate tissue morphogenesis in Arabidopsis thaliana. Development. 2014,141(21):4139-4148.

24. Kim CY, Koo YD, Jin JB, Moon BC, Kang CH, Kim ST, Park BO, Lee SY, Kim ML, Hwang I. Rice C2-domain proteins are induced and translocated to the plasma membrane in response to a fungal elicitor. Biochemistry (Mosc). 2003,42(40):11625-11633.

25. Yokotani N, Ichikawa T, Kondou Y, Maeda S, Iwabuchi M, Mori M, Hirochika H, Matsui M, Oda K. Overexpression of a rice gene encoding a small C2 domain protein OsSMCP1 increases tolerance to abiotic and biotic stresses in transgenic Arabidopsis. Plant Mol Biol. 2009,71(4):391-402.

26. Fu S, Fu L, Zhang X, Huang J, Yang G, Wang Z, Liu Y-G, Zhang G, Wu D, Xia J. OsC2DP, a novel C2 domain-containing protein is required for salt tolerance in rice. Plant Cell Physiol. 2019,60(10):2220-2230.

27. Liu L, Li C, Liang Z, Yu H. Characterization of multiple C2 domain and transmembrane region proteins in Arabidopsis. Plant Physiol. 2018,176(3):21192132.

28. Hao P, Wang H, Ma L, Wu A, Chen P, Cheng S, Wei H, Yu S. Genome-wide identification and characterization of multiple C2 domains and transmembrane region proteins in Gossypium hirsutum. BMC Genomics. 2020,21(1):1-16.

29. Huang R, Zhao J, Liu J, Wang Y, Han S, Zhao H. Genome-wide analysis and expression profiles of NTMC2 family genes in Oryza sativa. Gene. 2017,637:130-137.

30. Hammoudi V, Vlachakis G, Schranz ME, van den Burg HA. Whole-genome duplications followed by tandem duplications drive diversification of the protein modifier SUMO in Angiosperms. New Phytol. 2016,211(1):172-185.

Page $14 / 21$ 
31. Chow C-N, Chiang-Hsieh Y-F, Chien C-H, Zheng H-Q, Lee T-Y, Wu N-Y, Tseng K-C, Hou P-F, Chang W-C. Delineation of condition specific Cis-and Trans-acting elements in plant promoters under various Endo-and exogenous stimuli. BMC Genomics. 2018,19(2):109-121.

32. Lu X, Song S, Xiao Y, Fan F, Zhou Y, Jia G, Tang W, Peng J. Circadian clock-coordinated response to chilling stress in rice. Environ Exp Bot. 2021,185:104398.

33. Zheng L, Zhang C, Shi C, Wang Y, Zhou T, Sun F, Wang H, Zhao S, Qin Q, Qiao R. Rice stripe virus NS3 protein regulates primary miRNA processing through association with the miRNA biogenesis factor OsDRB1 and facilitates virus infection in rice. PLoS Pathog. 2017,13(10):e1006662.

34. Archak S, Nagaraju J. Computational prediction of rice (Oryza sativa) miRNA targets. Genomics Proteomics Bioinformatics. 2007,5(3-4):196-206.

35. Chinnusamy V, Ohta M, Kanrar S, Lee B-h, Hong X, Agarwal M, Zhu J-K. ICE1: a regulator of cold-induced transcriptome and freezing tolerance in Arabidopsis. Genes Dev. 2003,17(8):1043-1054.

36. Lee SK, Hong W-J, Silva J, Kim E-J, Park SK, Jung K-H, Kim Y-J. Global Identification of ANTH Genes Involved in Rice Pollen Germination and Functional Characterization of a Key Member, OsANTH3. Frontiers in Plant Science. 2021,12:637.

37. Zhang J-B, Wang X-P, Wang Y-C, Chen Y-H, Luo J-W, Li D-D, Li X-B. Genome-wide identification and functional characterization of cotton (Gossypium hirsutum) MAPKKK gene family in response to drought stress. BMC Plant Biol. 2020,20:1-14.

38. Nalefski EA, Wisner MA, Chen JZ, Sprang SR, Fukuda M, Mikoshiba K, Falke JJ. C2 domains from different Ca2 + signaling pathways display functional and mechanistic diversity. Biochemistry (Mosc). 2001,40(10):3089-3100.

39. Zang A, Xu X, Neill S, Cai W. Overexpression of OsRAN2 in rice and Arabidopsis renders transgenic plants hypersensitive to salinity and osmotic stress. J Exp Bot. 2010,61(3):777-789.

40. Giri J, Vij S, Dansana PK, Tyagi AK. Rice A20/AN1 zinc-finger containing stress-associated proteins (SAP1/11) and a receptor-like cytoplasmic kinase (OsRLCK253) interact via A20 zinc-finger and confer abiotic stress tolerance in transgenic Arabidopsis plants. New Phytol. 2011,191(3):721-732.

41. Leister D. Tandem and segmental gene duplication and recombination in the evolution of plant disease resistance genes. Trends Genet. 2004,20(3):116122.

42. Liu C, Wu Y, Liu Y, Yang L, Dong R, Jiang L, Liu P, Liu G, Wang Z, Luo L. Genome-wide analysis of tandem duplicated genes and their contribution to stress resistance in pigeonpea (Cajanus cajan). Genomics. 2021,113(1):728-735.

43. Yu J, Ke T, Tehrim S, Sun F, Liao B, Hua W. PTGBase: an integrated database to study tandem duplicated genes in plants. Database. $2015,2015$.

44. Clark RM, Schweikert G, Toomajian C, Ossowski S, Zeller G, Shinn P, Warthmann N, Hu TT, Fu G, Hinds DA. Common sequence polymorphisms shaping genetic diversity in Arabidopsis thaliana. Science. 2007,317(5836):338-342.

45. Rizzon C, Ponger L, Gaut BS. Striking similarities in the genomic distribution of tandemly arrayed genes in Arabidopsis and rice. PLoS Comput Biol. 2006,2(9):e115.

46. Bellieny-Rabelo D, Oliveira AEA, Venancio TM. Impact of whole-genome and tandem duplications in the expansion and functional diversification of the Fbox family in legumes (Fabaceae). PLoS ONE. 2013,8(2):e55127.

47. Yang W-Q, Lai Y, Li M-N, Xu W-Y, Xue Y-B. A novel C2-domain phospholipid-binding protein, OsPBP1, is required for pollen fertility in rice. Mol Plant. 2008,1(5):770-785

48. Jiang SY, Cai M, Ramachandran S. The Oryza sativa no pollen (Osnop) gene plays a role in male gametophyte development and most likely encodes a C2-GRAM domain-containing protein. Plant Mol Biol. 2005,57(6):835-853.

49. Liu C, Ou S, Mao B, Tang J, Wang W, Wang H, Cao S, Schläppi MR, Zhao B, Xiao G. Early selection of bZIP73 facilitated adaptation of japonica rice to cold climates. Nat Commun. 2018,9(1):1-12.

50. Liu C, Schläppi MR, Mao B, Wang W, Wang A, Chu C. The bZIP 73 transcription factor controls rice cold tolerance at the reproductive stage. Plant Biotechnol J. 2019,17(9):1834-1849.

51. Fjell CD, Jenssen H, Fries P, Aich P, Griebel P, Hilpert K, Hancock RE, Cherkasov A. Identification of novel host defense peptides and the absence of adefensins in the bovine genome. Proteins: Struct, Funct, Bioinf. 2008,73(2):420-430.

52. Chen $\mathrm{C}$, Chen $\mathrm{H}$, Zhang Y, Thomas HR, Frank MH, He Y, Xia R. TBtools: an integrative toolkit developed for interactive analyses of big biological data. Mol Plant. 2020,13(8):1194-1202.

53. Yu CS, Chen YC, Lu CH, Hwang JK. Prediction of protein subcellular localization. Proteins: Struct, Funct, Bioinf. 2006,64(3):643-651.

54. Kumar S, Stecher G, Li M, Knyaz C, Tamura K. MEGA X: molecular evolutionary genetics analysis across computing platforms. Mol Biol Evol. 2018,35(6):1547-1549.

55. Letunic I, Bork P. Interactive Tree Of Life (iTOL) v4: recent updates and new developments. Nucleic Acids Res. 2019,47(W1):W256-W259.

56. Hu B, Jin J, Guo A-Y, Zhang H, Luo J, Gao G. GSDS 2.0: an upgraded gene feature visualization server. Bioinformatics. 2015,31(8):1296-1297.

57. Larkin MA, Blackshields G, Brown NP, Chenna R, McGettigan PA, McWilliam H, Valentin F, Wallace IM, Wilm A, Lopez R. Clustal W and Clustal X version 2.0. Bioinformatics. 2007,23(21):2947-2948.

58. Stothard P. The sequence manipulation suite: JavaScript programs for analyzing and formatting protein and DNA sequences. Biotechniques. 2000,28(6):1102-1104.

59. Altschul SF, Gish W, Miller W, Myers EW, Lipman DJ. Basic local alignment search tool. J Mol Biol. 1990,215(3):403-410.

60. Wang Y, Tang H, DeBarry JD, Tan X, Li J, Wang X, Lee T-h, Jin H, Marler B, Guo H. MCScanX: a toolkit for detection and evolutionary analysis of gene synteny and collinearity. Nucleic Acids Res. 2012,40(7):e49-e49. 
61. Cui L, Yang G, Yan J, Pan Y, Nie X. Genome-wide identification, expression profiles and regulatory network of MAPK cascade gene family in barley. BMC Genomics. 2019,20(1):1-20.

62. Krzywinski M, Schein J, Birol I, Connors J, Gascoyne R, Horsman D, Jones SJ, Marra MA. Circos: an information aesthetic for comparative genomics. Genome Res. 2009,19(9):1639-1645.

63. Wang D, Zhang Y, Zhang Z, Zhu J, Yu J. KaKs_Calculator 2.0: a toolkit incorporating gamma-series methods and sliding window strategies. Genomics Proteomics Bioinformatics. 2010,8(1):77-80.

64. Wang L, Xie W, Chen Y, Tang W, Yang J, Ye R, Liu L, Lin Y, Xu C, Xiao J. A dynamic gene expression atlas covering the entire life cycle of rice. The Plant Journal. 2010,61(5):752-766.

65. Garg R, Tyagi AK, Jain M. Microarray analysis reveals overlapping and specific transcriptional responses to different plant hormones in rice. Plant signaling \& behavior. 2012,7(8):951-956.

66. Sato Y, Takehisa H, Kamatsuki K, Minami H, Namiki N, Ikawa H, Ohyanagi H, Sugimoto K, Antonio BA, Nagamura Y. RiceXPro version 3.0: expanding the informatics resource for rice transcriptome. Nucleic Acids Res. 2013,41(D1):D1206-D1213.

67. Lescot M, Déhais P, Thijs G, Marchal K, Moreau Y, Van de Peer Y, Rouzé P, Rombauts S. PlantCARE, a database of plant cis-acting regulatory elements and a portal to tools for in silico analysis of promoter sequences. Nucleic Acids Res. 2002,30(1):325-327.

68. Rao X, Huang X, Zhou Z, Lin X. An improvement of the $2^{\wedge}$ (-delta delta CT) method for quantitative real-time polymerase chain reaction data analysis. Biostatistics, bioinformatics and biomathematics. 2013,3(3):71.

69. Dai X, Zhao PX. psRNATarget: a plant small RNA target analysis server. Nucleic Acids Res. 2011,39(suppl_2):W155-W159.

70. Shannon P, Markiel A, Ozier O, Baliga NS, Wang JT, Ramage D, Amin N, Schwikowski B, Ideker T. Cytoscape: a software environment for integrated models of biomolecular interaction networks. Genome Res. 2003,13(11):2498-2504.

71. Yi X, Du Z, Su Z. PlantGSEA: a gene set enrichment analysis toolkit for plant community. Nucleic Acids Res. 2013,41(W1):W98-W103.

72. Kim T-S, He Q, Kim K-W, Yoon M-Y, Ra W-H, Li FP, Tong W, Yu J, Oo WH, Choi B. Genome-wide resequencing of KRICE_CORE reveals their potential for future breeding, as well as functional and evolutionary studies in the post-genomic era. BMC Genomics. 2016,17(1):1-13.

73. Zhang H, San ML, Jang S-G, Lee J-H, Kim N-E, Lee A-R, Park S-Y, Cao F-Y, Chin J-H, Kwon S-W. Genome-Wide Association Study of Root System Development at Seedling Stage in Rice. Genes. 2020,11(12):1395.

74. Zhang H, Wu T, Li Z, Huang K, Kim N-E, Ma Z, Kwon S-W, Jiang W, Du X. OsGATA16, a GATA Transcription Factor, Confers Cold Tolerance by Repressing OsWRKY45-1 at the Seedling Stage in Rice. Rice. 2021,14(1):1-15.

75. Leigh JW, Bryant D. popart: full-feature software for haplotype network construction. Methods in Ecology and Evolution. 2015,6(9):1110-1116.

76. Barrett JC, Fry B, Maller J, Daly MJ. Haploview: analysis and visualization of LD and haplotype maps. Bioinformatics. 2005,21(2):263-265.

\section{Figures}

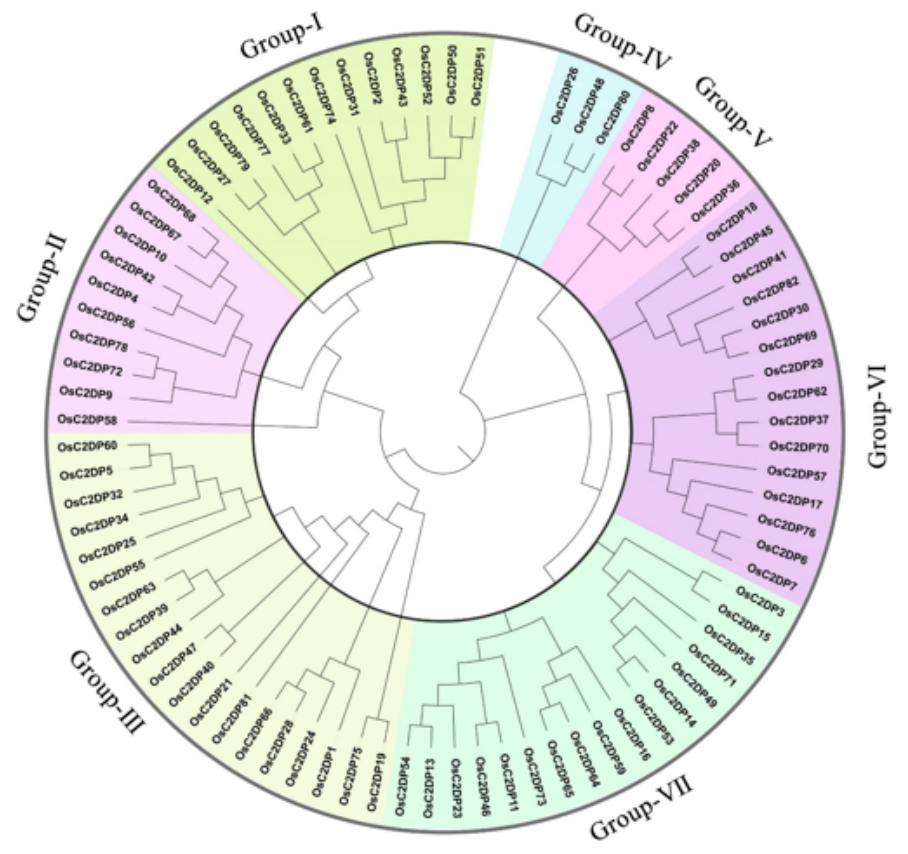

Figure 1

Phylogenetic tree of OsC2DPs in rice genome. Different colors represent different groups 
A

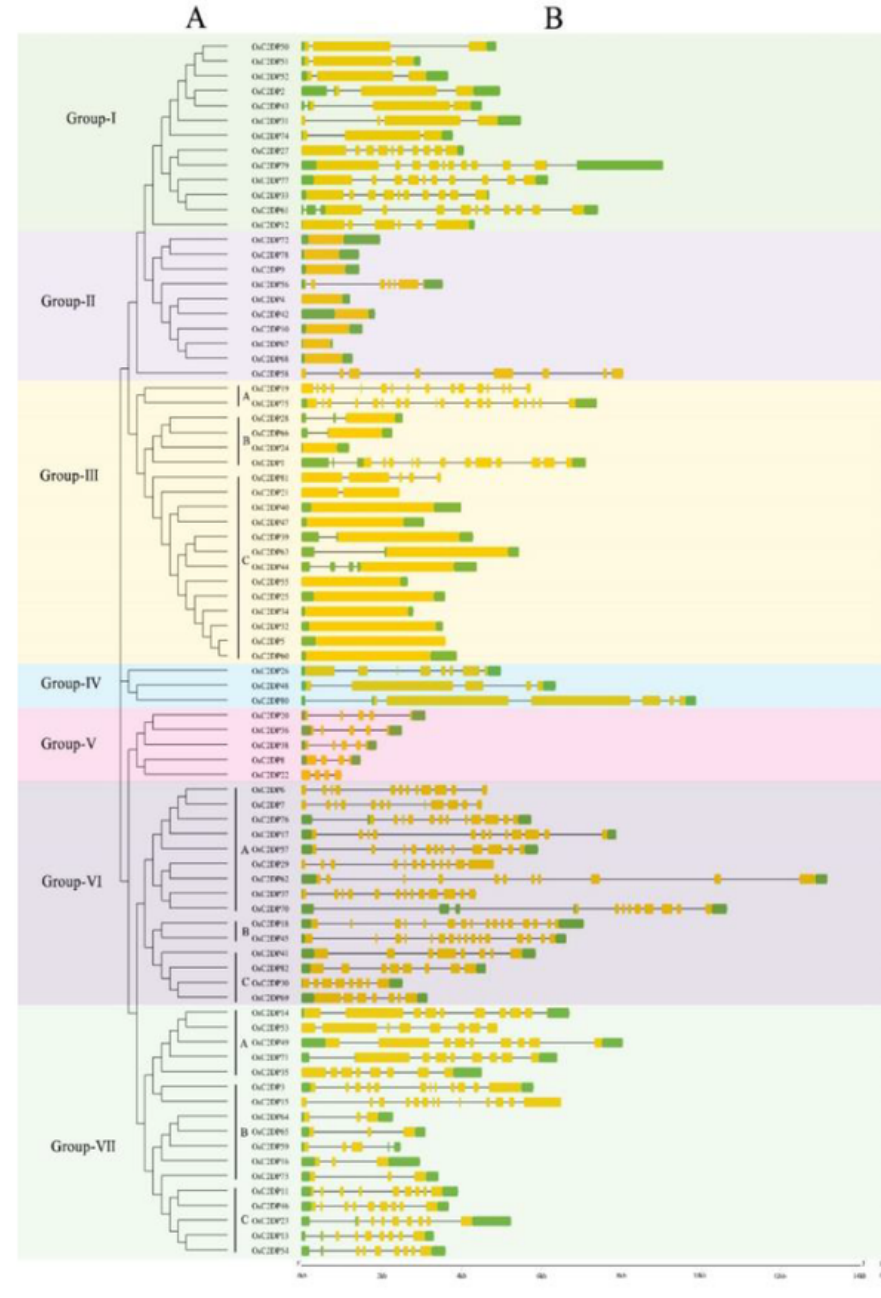

C

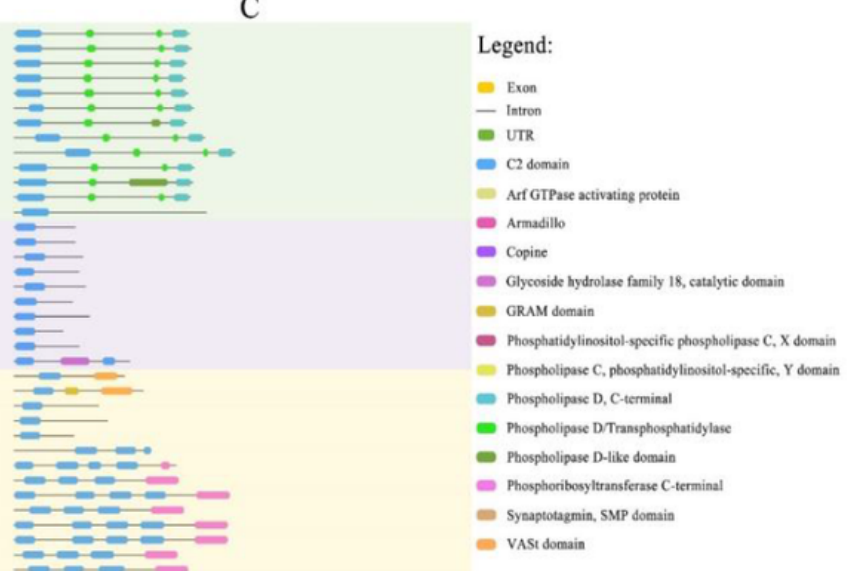

\section{Figure 2}

Structural schematic diagram of the OsC2DP gene family. a Phylogenetic relationship of OsC2DPs. b Gene structure of OsC2DPs. c Putative domain structure of OsC2DPs 


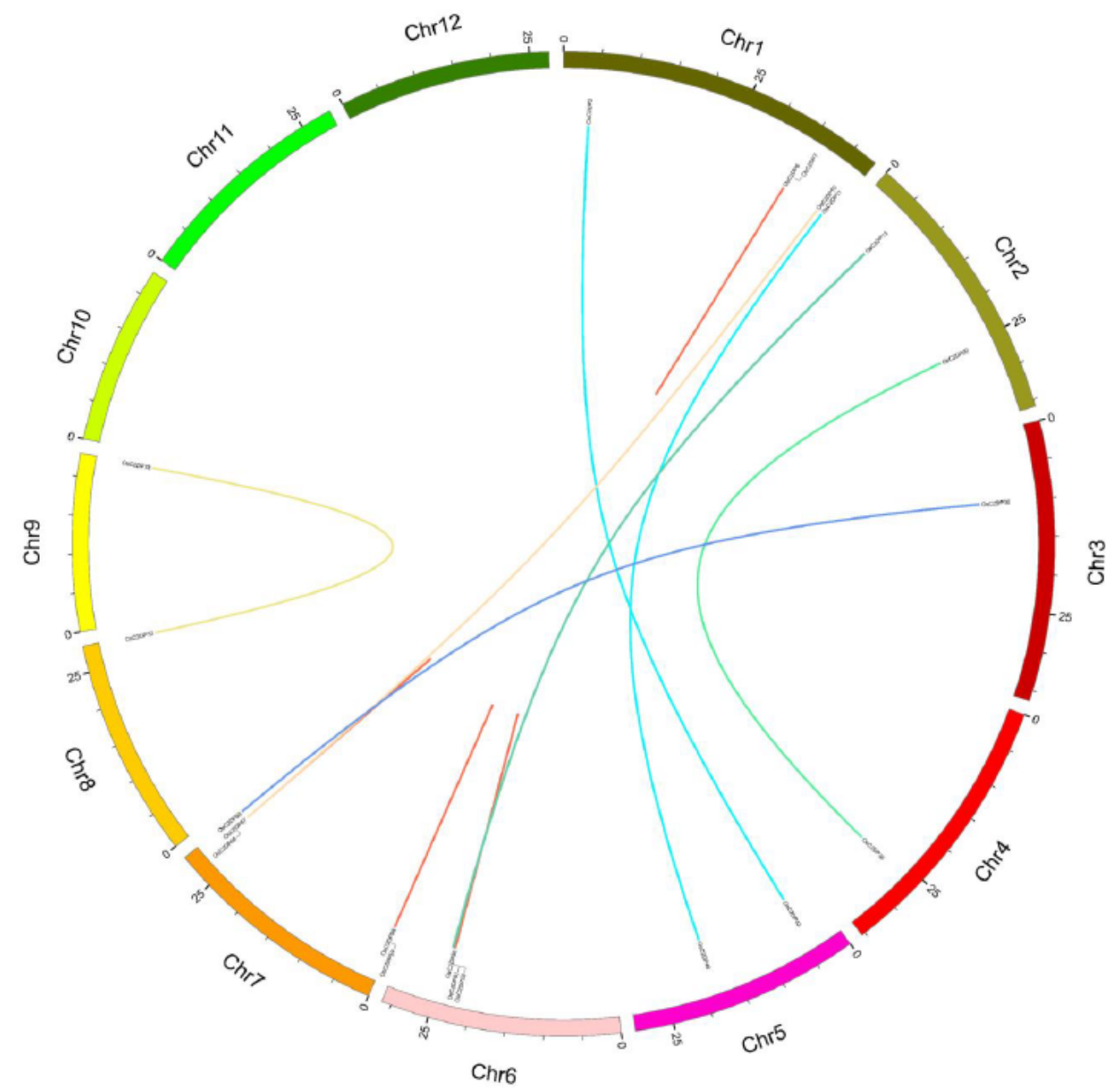

Figure 3

Synteny analysis of OsC2DPs intra rice genome. Each rice chromosome is displayed in a different color. Duplicated gene pairs are displayed and linked using colored lines; red half lines represent tandem duplications 


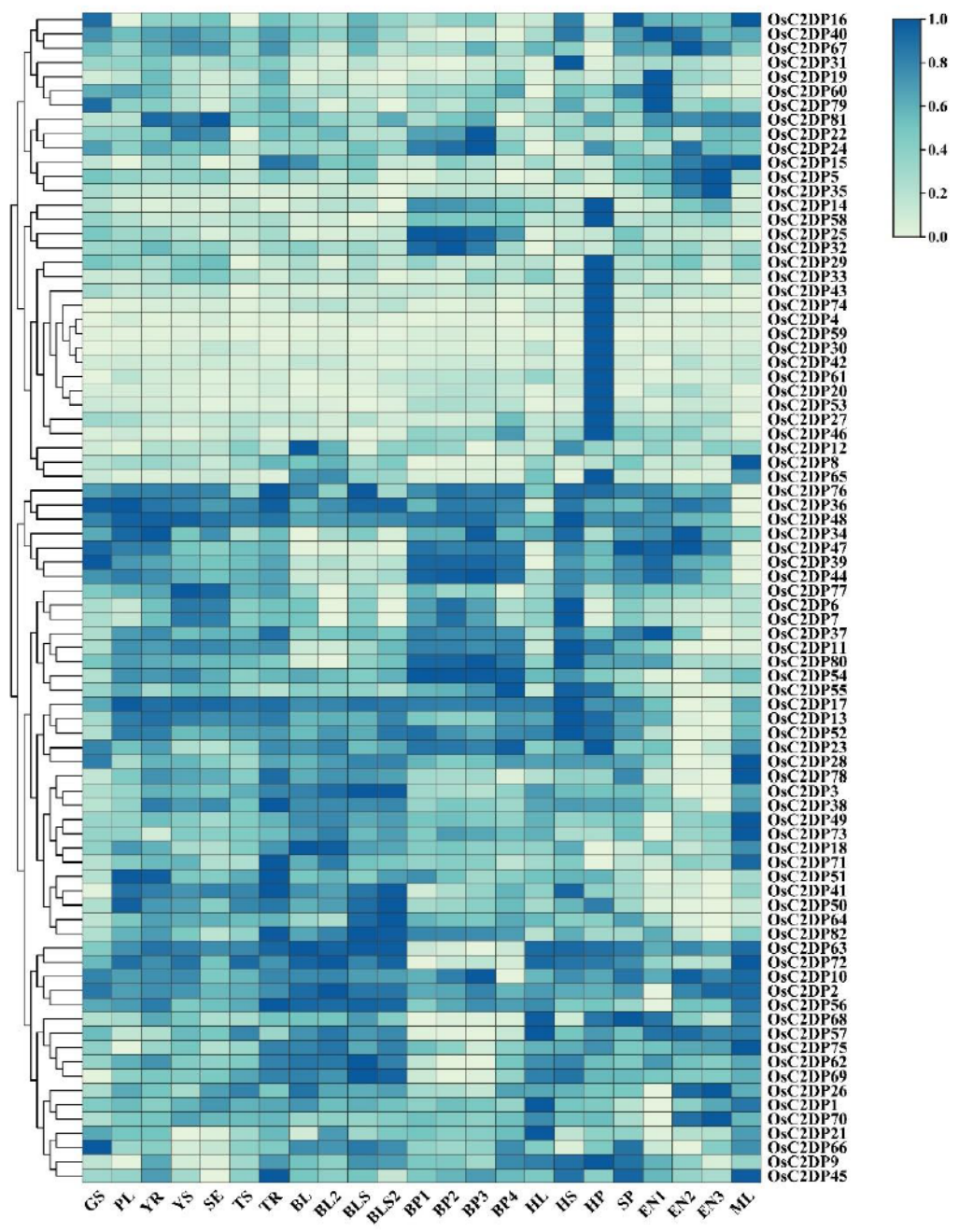

\section{Figure 4}

Expression profiles of OsC2DPs in diverse tissues across different stages. GS: germinating seed; PL: plumule; YR: radicle; YS: young seedling; SE: seedling at trefoil stage; TS: shoot under 2 tillers; TR: root under 2 tillers; BL: mature leaf blade under young panicle; BL2: mature leaf blade under mature panicle; BLS: mature leaf sheath under young panicle; BLS2: mature leaf sheath under mature panicle; BP1: developing panicle (length < $1 \mathrm{~mm}$ ); BP2: developing panicle (3 $\mathrm{mm}<$ length < $5 \mathrm{~mm})$; BP3: developing panicle $(10 \mathrm{~mm}<$ length < $15 \mathrm{~mm})$; BP4: developing panicle $(40 \mathrm{~mm}<$ length < $50 \mathrm{~mm})$; $\mathrm{HL}$ : flag leaf in heading date; HS: stem in heading stage; HP: panicle in heading stage; SP: spikelet; EN1: endosperm under 7 days after pollination; EN2: endosperm under 14 days after pollination; EN3: endosperm under 21 days after pollination; and ML: flag leaf under mature stage 

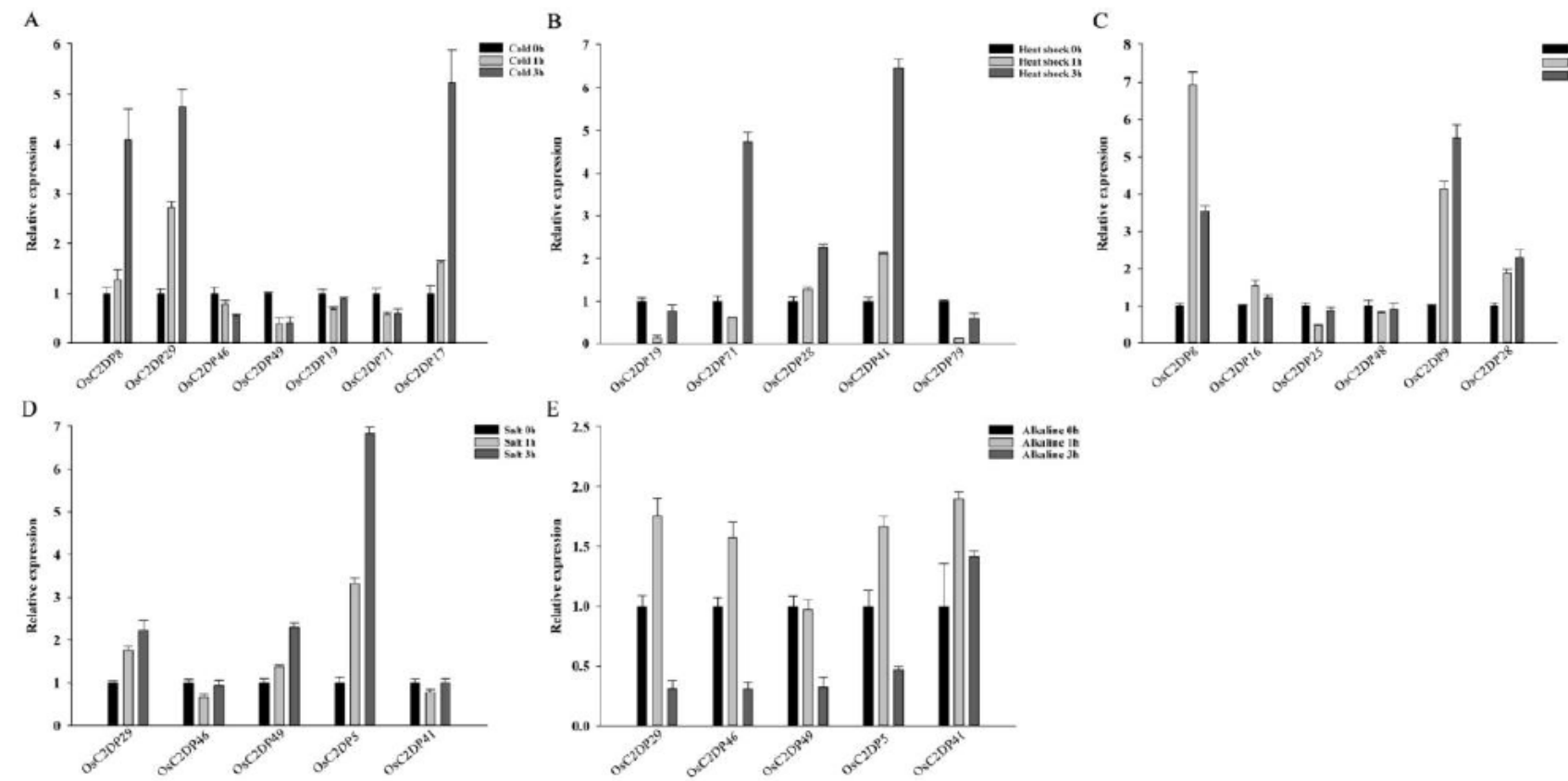

\section{Figure 5}

Time-course expression analysis of OsC2DPs after exposure to abiotic stress. a Cold. b Heat shock. c Drought. d Salt. e Alkaline. Data represent the mean \pm SE of three replicates

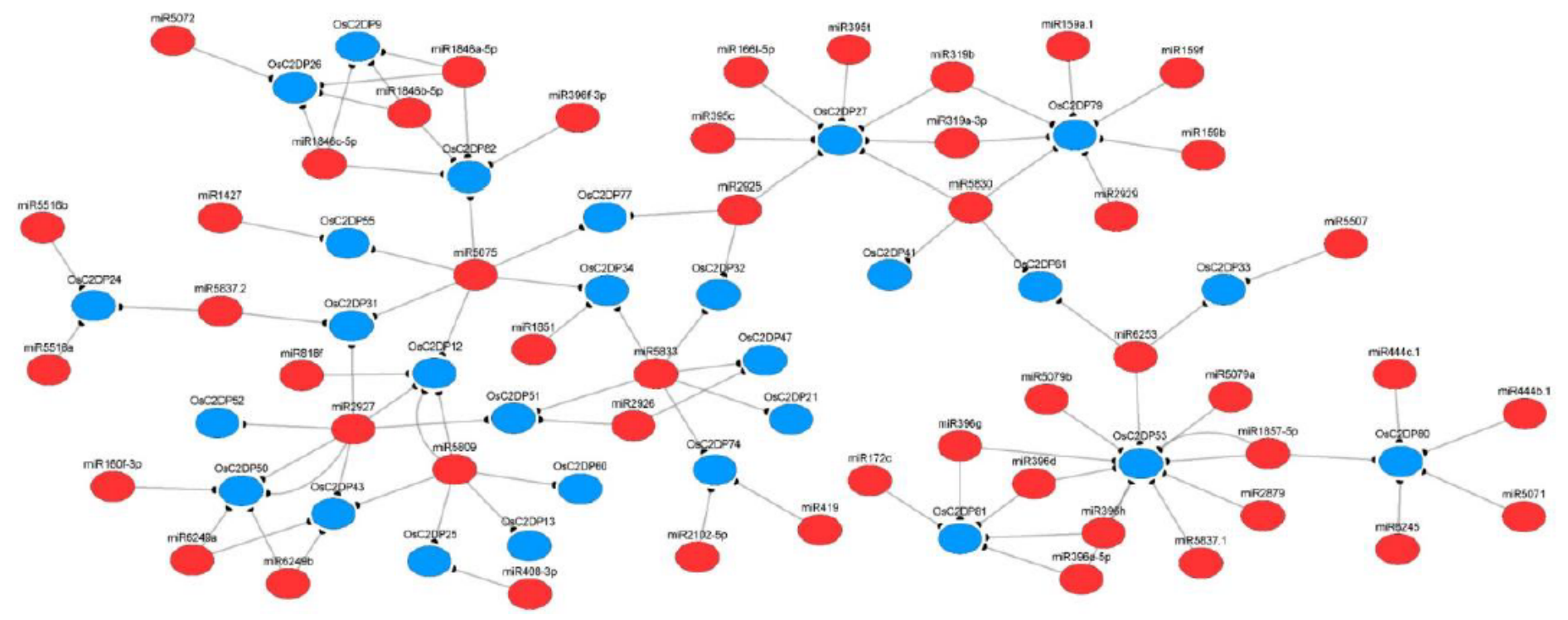

Figure 6

Putative regulation network for miRNA-targets of OsC2DPs. The predicted targets are connected by solid lines 
A

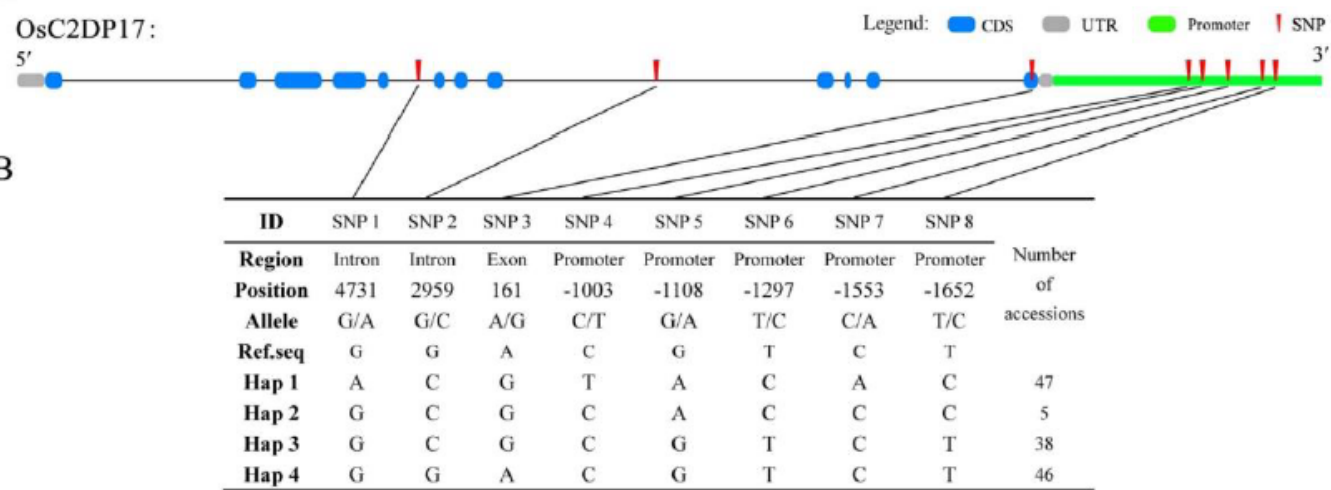

C

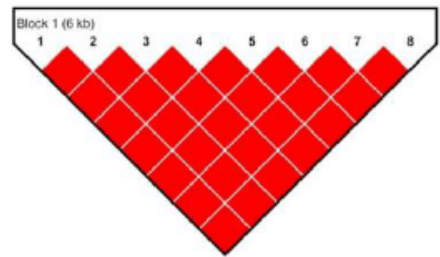

E

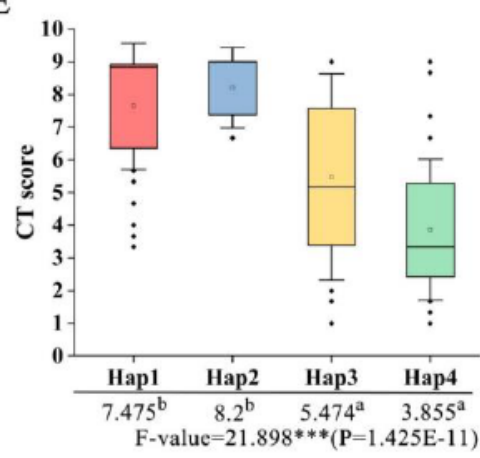

$\mathrm{D}$

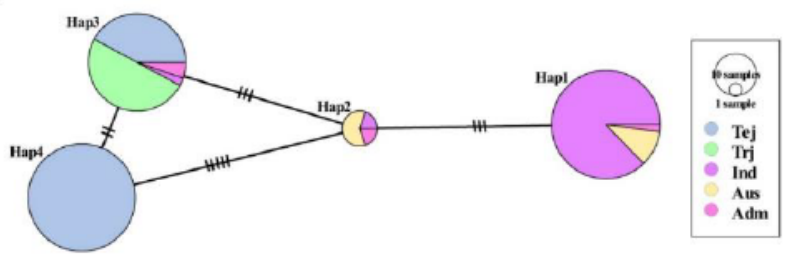

$\mathrm{F}$

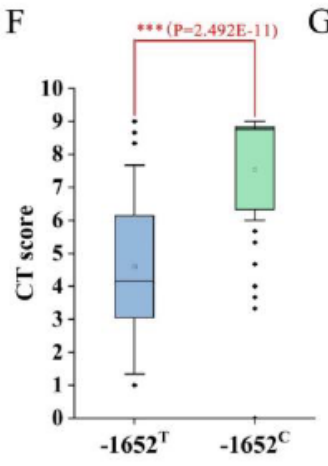

G

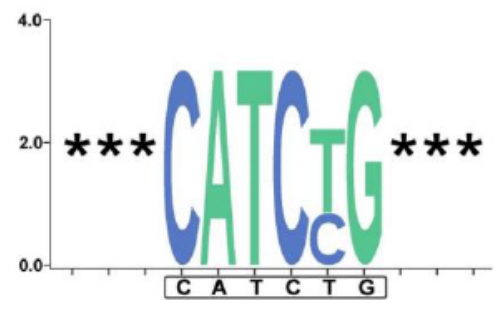

\section{Figure 7}

Haplotype analysis of OsC2DP17. a Structural representation of OsC2DP17 and the upstream promoter region. b LD analysis of OsC2DP17 using SNPs in a; pure red squares represent complete linkage level between each SNP. c OsC2DP17 SNPs and haplotype groups in 137 rice accessions. SNP positions are given relative to the start of the 5' UTR. Hap: Haplotype. d Haplotype network variation of OsC2DP17. Circle size represents the number of accessions in each Hap, and the number of transverse lines between each Hap represents the number of nucleotide variations. Tej: Temperate Japonica; Trj: Tropical Japonica; Ind: Indica; and Adm: Admixture rice varieties. e Association of the CT score with haplotype. $f$ SNP haplotype analysis for the CT score. Superscripts T and C in $f$ indicate the $\mathrm{T}$ and $\mathrm{C}$ genotypes in the present accession panel. g SeqLogo plot for cold-related motif MYCCONSENSUSAT (CATCTG). Size of letters represent genotype frequency in the present accession panel. Different letters indicate significant differences among haplotypes (ANOVA, Duncan test), asterisks indicate significant differences in phenotype between genotypes (Student's t-test, ${ }^{\star \star \star} p<0.001$ )

\section{Supplementary Files}

This is a list of supplementary files associated with this preprint. Click to download.

- UploadSupplementFigandTable.zip 